\title{
Monoterpenoid indole alkaloids biosynthesis and its regulation in Catharanthus roseus: a literature review from genes to metabolites
}

\author{
Qifang Pan (1) - Natali Rianika Mustafa • \\ Kexuan Tang • Young Hae Choi • \\ Robert Verpoorte
}

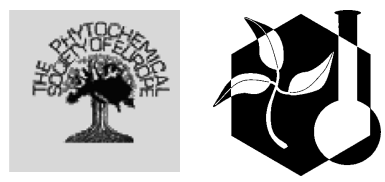

Received: 8 December 2014 / Accepted: 25 March 2015/Published online: 1 April 2015

(C) Springer Science+Business Media Dordrecht 2015

\begin{abstract}
As the only source for the low-abundance antitumor agents vinblastine and vincristine, Catharanthus roseus is highly valued and has been studied extensively as a model for medicinal plants improvement. The biosynthesis of these monoterpenoid indole alkaloids (MIAs) is a complex multistep enzymatic network that is tightly regulated by developmental and environmental factors. Here we review the knowledge achieved in the past 30 years of the MIA pathway in $C$. roseus, from genetic to metabolic aspects. Two early precursor pathways and a late mono-/bis-indole alkaloid pathway have been largely elucidated and established, as well as their intercellular and subcellular compartmentation. Many genes encoding constitutive structural biosynthetic enzymes, transcription factors, and transporters involved in these pathways have been cloned, characterized and applied in metabolic engineering strategies to improve the MIA production. However, genetic modification in the pathway in $C$.
\end{abstract}

Q. Pan $(\bowtie) \cdot K$. Tang

Plant Biotechnology Research Center, SJTU-Cornell Institute of Sustainable Agriculture and Biotechnology, Fudan-SJTU-Nottingham Plant Biotechnology R\&D

Center, School of Agriculture and Biology, Shanghai

Jiaotong University, Dongchuan Road 800,

Shanghai 200240, China

e-mail: pqfang2013@aliyun.com; panqf@sjtu.edu.cn

N. R. Mustafa · Y. H. Choi · R. Verpoorte

Natural Products Laboratory, Institute of Biology Leiden, Leiden University, Sylviusweg 72, 2333 BE Leiden,

The Netherlands roseus resulted in complicated changes of both secondary and primary metabolism, affecting not only the MIA pathway but also other pathways. Research at metabolic level is required to increase the knowledge on the genetic regulation of the whole metabolic network connected to the MIA biosynthesis. Nuclear magnetic resonance-based metabolomics (metabolic profiling, fingerprinting and flux analyses) in combination with other "omics" have been implemented in studies of $C$. roseus for pathway elucidation, including among others, understanding stress response, cross talk between pathways, and diversion of carbon fluxes, with the aim to fully unravel the MIA biosynthesis, its regulation and the function of the alkaloids in the plant from a systems biology point of view.

Keywords Catharanthus roseus $\cdot$ Monoterpenoid indole alkaloids · Metabolic engineering $\cdot$ NMR . Metabolomics

\section{Introduction}

Monoterpenoid indole alkaloids (MIAs) form a major group of alkaloids in the plant kingdom, comprising of over 3000 identified alkaloids to date. The MIAs have structures derived from strictosidine formed from tryptamine and $\mathrm{C}_{10}$ part from the iridoid secologanin. Many of these natural products are physiologically active in mammals. But they are found to be confined 
to eight different plant families (e.g., the most important ones: Apocynaceae, Loganiaceae, and $\mathrm{Ru}-$ biaceae.). Several pharmaceuticals belonging to the MIA group are commercially isolated from plant materials (Table 1), like the antimalarial alkaloid quinine from Cinchona officinalis, the antineoplastic camptothecine from Camptotheca acuminata, the rat poison and tonic strychnine from Strychnos nuxvomica, the antihypertensive and tranquilizer reserpine from Rauvolfia species and the antitumor agents vinblastine and vincristine from Catharanthus roseus (periwinkle) (Kutchan 1995).

Catharanthus roseus, belonging to the Apocynaceae family, is a medical plant of great pharmaceutical interest for its capacity to biosynthesize a great variety of MIAs (>130), which have a high economic value due to their wide spectrum of pharmaceutical applications. Besides the most well-known bisindole alkaloids (vinblastine and vincristine), C. roseus also produces ajmalicine used as antihypertensive and serpentine used as sedative. The very small amounts of the dimeric alkaloids in C. roseus and the difficulty of their extraction and purification explain the high costs of these MIAs. Although total chemical synthesis of these complex alkaloids is of academic interest, this is not likely to be applied commercially due to the low yields. However, the dimerization reaction coupling vindoline and catharanthine, which in the plant is catalyzed by a peroxidase, has been mimicked chemically and is now used to couple the much more abundant monomers. The in vitro production systems using plant cell cultures or hairy roots of $C$. roseus have been developed but failed to synthesize vindoline, one of the precursors needed for the bisindole alkaloids. To develop novel sources of these compounds, e.g. by synthetic biology, requires thorough knowledge of all genes, enzymes, and intermediates in the MIA biosynthetic pathway and the regulation mechanism behind it.

In the past decades there have been extensive efforts and in-depth studies on MIA biosynthesis in C. roseus by numerous groups of researchers all across the globe. Nowadays the "Omics" tools, such as genomics, transcriptomics, proteomics and metabolomics, can provide us with enormous amounts of information about the genes, enzymes, transcription factors, intermediates, pathways, and compartmentation of MIA biosynthesis in $C$. roseus cell cultures, hairy roots and plants. These tools will be very helpful to clarify some unresolved parts of the iridoid pathway, the catharanthine biosynthesis, transport, and the signal-transduction and regulation of the pathway via transcription factors like the octadecanoid-responsive Catharanthus AP2-domain (Orca's).

The latest developments in the studies of the biosynthesis of MIA and its regulation in C. roseus by genomic and metabolomic studies are reviewed in the present update.

Table 1 Active MIAs, their functions and plants that produce them

\begin{tabular}{lll}
\hline Alkaloids & Function & Plants \\
\hline Ajmalicine & Antihypertensive & Catharanthus roseus \\
Camptothecine & Antineoplastic & Camptotheca acuminata \\
Ellipticine & Antitumour & Ochrosia elliptica \\
Emetine & Anti-protozoal & Carapichea ipecacuanha \\
Quinidine & Class I antiarrhythmic agent (Ia) in the heart & Cinchona spp. \\
Quinine & Antimalarial alkaloid & Cinchona spp. \\
Rescinnamine & Antihypertensive & Rauwolfia spp. \\
Reserpine & Antipsychotic, antihypertensive & Rauwolfia serpentina \\
Serpentine & Sedative & Catharanthus roseus \\
Strychnine & Rat poison and homeopathic & Strychnos nux-vomica \\
Toxiferine & Curare toxin, muscle relaxant & Strychnos toxifera \\
Vinblastine, vincristine & Antitumor & Catharanthus roseus \\
Vincamine & Peripheral vasodilator that increases blood flow to the brain & Vinca minor \\
Yohimbine & Mild monoamine oxidase inhibitors with stimulant and aphrodisiac effects & Pausinystaliayohimbe \\
\hline
\end{tabular}




\section{Alkaloid biosynthesis and its compartmentation in Catharanthus roseus}

MIA biosynthesis in $C$. roseus is a complex pathway including at least 30 coordinately regulated enzymatic steps producing at least 35 known intermediates. The pathway is spread over at least four different cell types and in these cells at least five different subcellular compartments are involved, which means that transport is a major rate determining factor, which besides physical chemical principles (e.g. diffusion, mass transfer between two phases) also involves different types of active selective transporter proteins (Courdavault et al. 2014; Roytrakul and Verpoorte 2007). Up to now, 30 biosynthetic and 4 types of regulatory genes from the MIA pathways have been cloned and identified. However, there are still unknown parts of MIA biosynthesis to be solved. Alkaloids in C. roseus derive from the convergence of two primary metabolic routes, i.e. the shikimate and the secoiridoid pathways that respectively provide the indole and the terpene moiety to the basic backbone as found in strictosidine (van der Heijden et al. 2004; Verma et al. 2012).

The shikimate-chorismate-indole pathway and its localization

The shikimate pathway, a major biosynthetic route for both primary and secondary metabolism, starts with phosphoenolpyruvate and erythrose-4-phosphate and ends with chorismate (Herrmann and Weaver 1999) (Fig. 1). Chorismate is an important starting point and the substrate of 5 enzymes that are the gatekeepers of the five pathways that branch from chorismate: anthranilate synthase (AS) on the branch leading to tryptophan; chorismate mutase $(\mathrm{CM})$ on the branch leading to phenylalanine and tyrosine; isochorismate synthase (ICS) on the branch leading to isochorismate, salicylate and 2,3-dihydrobenzoic acid (2,3-DHBA); chorismate pyruvate-lyase synthase (CPL) on the branch to p-hydroxybenzoate (a precursor of shikonin); and p-aminobenzoate synthase on the branch to folates (Mustafa and Verpoorte 2007).

The anthranilate pathway leads to the formation of the aromatic amino acid tryptophan, which provides the indole moiety to MIAs (Fig. 1). Anthranilate synthase (AS) is the first key enzyme in the synthesis of tryptophan and indole-3-acetic acid, which consists of two large ( $\alpha$-subunits) and two small ( $\beta$-subunits) subunits (Poulsen et al. 1993). The $\alpha$-subunit of AS is responsible for catalyzing the conversion of chorismate into anthranilate and is sensitive to tryptophanmediated feedback inhibition (Verpoorte et al. 1997; Radwanski and Last 1995). But after AS, none of the enzymes leading to tryptophan have been studied in $C$. roseus so far (El-Sayed and Verpoorte 2007). Tryptophan is conversed into tryptamine by tryptophan decarboxylase (TDC) (Noé et al. 1984). The genes of both AS (Hong et al. 2006) and TDC (Canel et al. 1998; Goddijn et al. 1995; Whitmer et al. 2002b) have been cloned and overexpressed in cell cultures or hairy roots of $C$. roseus.

In the aerial tissues, the epidermis of leaf harbors the maximum expression of the $T D C$ gene. In the underground tissues, $T D C$ transcripts are localized in protoderm and cortical cells around the root apical meristem (St-Pierre et al. 1999; Irmler et al. 2000). The early steps of the tryptophan pathway are thought to occur in plastids (Zhang et al. 2001). The TDC enzyme essentially operates in the cytosol (De Luca and Cutler 1987). This implies that tryptophan has to move out from plastids to the cytosol for its decarboxylation by TDC to yield tryptamine, which is immediately transported to the cell vacuole for its subsequent condensation with secologanin (Fig. 1). The leaf epidermis of $C$. roseus is also a site of expression of PAL, C4H and CHS genes of the phenylpropanoid pathway (Mahroug et al. 2006; Murata et al. 2008).

The MEP-secoiridoid pathway and its localization

The terpenoid moiety of MIAs comes from secologanin biosynthesized via the secoiridoid pathway, which derives from a universal precursor for all monoterpenes geranyl diphosphate (GPP). There are two separate pathways in plants to produce isopentenyl diphosphate (IPP), the central precursor of all isoprenoids. One is the mevalonate pathway leading to the formation of triterpenes (sterols) and certain sesquiterpenes (Newman and Chappell 1999; Lange and Croteau 1999). The other is the mevalonateindependent (MEP) pathway leading to the formation of monoterpenes, diterpenes, tetraterpenes (carotenoids) and the prenyl-sidechains of chlorophyll (Eisenreich et al. 1996; Arigoni et al. 1997; Rohmer 1999). ${ }^{13} \mathrm{C}$ labeling experiments followed by NMR proved that the MEP pathway is the major route for the 
Fig. 1 Shikimatechorismate-indole pathway and its compartmentation at intercellular and subcellular level. Solid arrows represent one-step reactions; broken arrows represent multiple or uncharacterized reactions. "? cell": unclear cellular localization

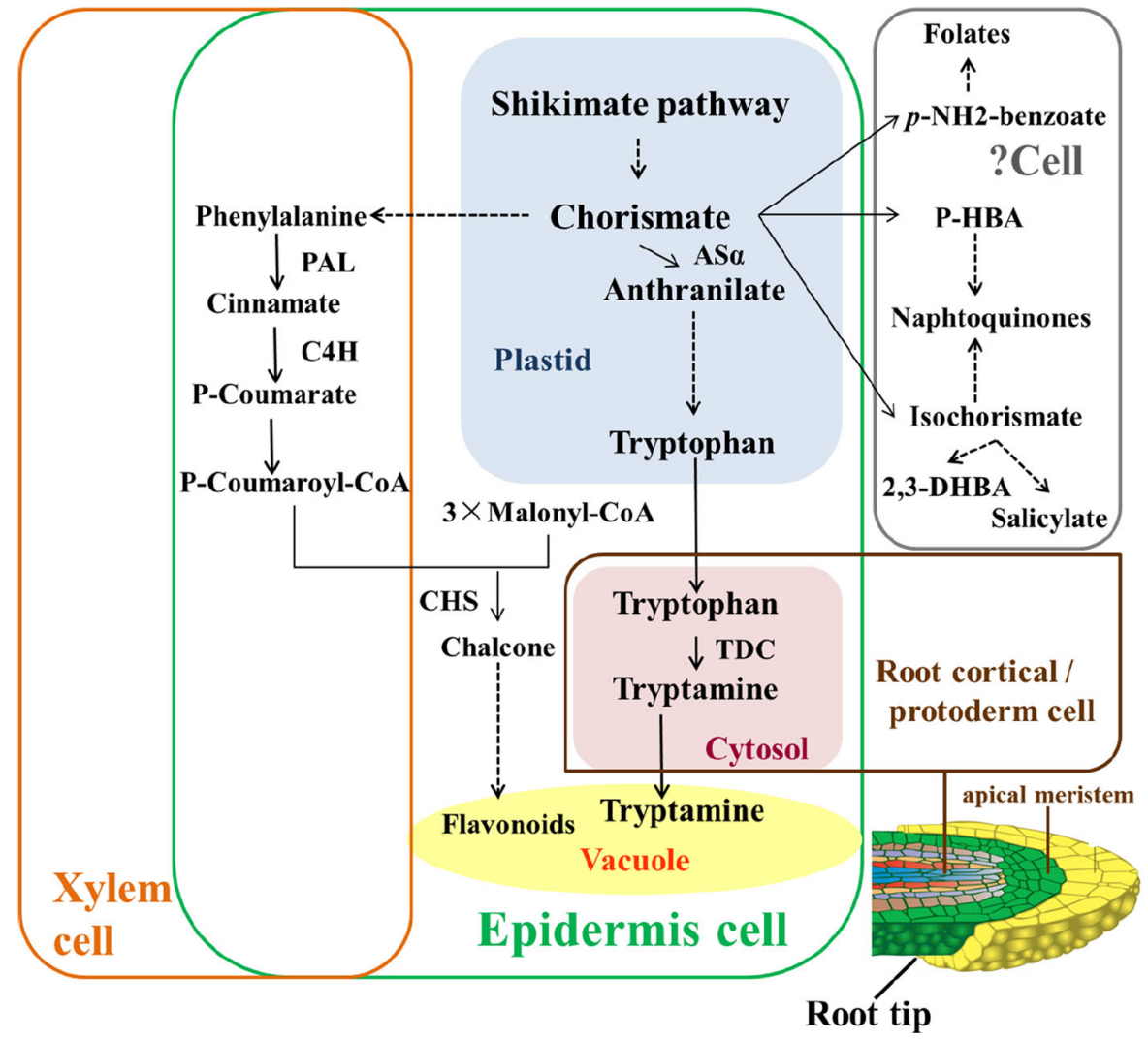

biosynthesis of secologanin in C. roseus (Contin et al. 1998).

The MEP pathway leading to the IPP formation has been unraveled in plants. A complete set of MEP pathway genes in Arabidopsis homologous to the corresponding genes in E. coli have been identified (Ganjewala et al. 2009). The MEP pathway involves 7 enzymatic steps, starting from the condensation of pyruvate with D-glyceraldehyde 3-phosphate ending with the production of IPP (El-Sayed and Verpoorte 2007). The pathway starts with the enzyme 1-deoxy-Dxylulose-5-phosphate synthase (DXS) that catalyzes the condensation of pyruvate with D-glyceraldehyde 3-phosphate to 1-deoxy-D-xylulose-5-phosphate, the first step of the MEP pathway. This product is then converted into 2-C-methyl-D-erythriol-4-phosphate by the enzyme 1-deoxy-D-xylulose-5-phosphate reducto isomerase (DXR) (Fig. 2).

The final product IPP is converted to dimethylallyl diphosphate (DMAPP) by isopentenyl diphosphate isomerase (IDI). The encoding gene was cloned from many sources including from $C$. roseus (CrIDIl)
(Guirimand et al. 2012). This enzyme, previously abbreviated as IPPI (Ramos-Valdivia et al. 1997), is expressed in all organs (roots, flowers and young leaves). CrIDII produces both long and short transcripts giving rise to the corresponding proteins with and without a $N$-terminal transit peptide (TP), respectively (Guirimand et al. 2012).

After IPP synthesis via the MEP pathway, a key step in the monoterpenoid branch towards the MIA pathway is the generation of GPP, the entry point to the formation of the monoterpene moiety, from the condensation of IPP and DMAPP by GPP synthase (GPPS) (Contin et al. 1998; Hedhili et al. 2007; Fig. 2), which belongs to the family of short-chain prenyltransferases. In $C$. roseus, three genes were found to encode proteins with sequence similarity to the large subunit (CrGPPS.LSU) and the small subunit (CrGPPS.SSU) of heteromeric GPPSs, and a homomeric GPPSs, respectively (Rai et al. 2013). CrGPPS.LSU is a bifunctional enzyme producing both GPP and geranylgeranyl diphosphate (GGPP). CrGPPS.SSU could function as a primary regulator to 
Fig. 2 MEP-secoiridoid pathway and its compartmentation at intercellular and subcellular level. Solid arrows represent one-step reactions; the broken arrow represents multiple reactions. IPAP, internal phloem-associated parenchyma. ER endoplasmic reticulum

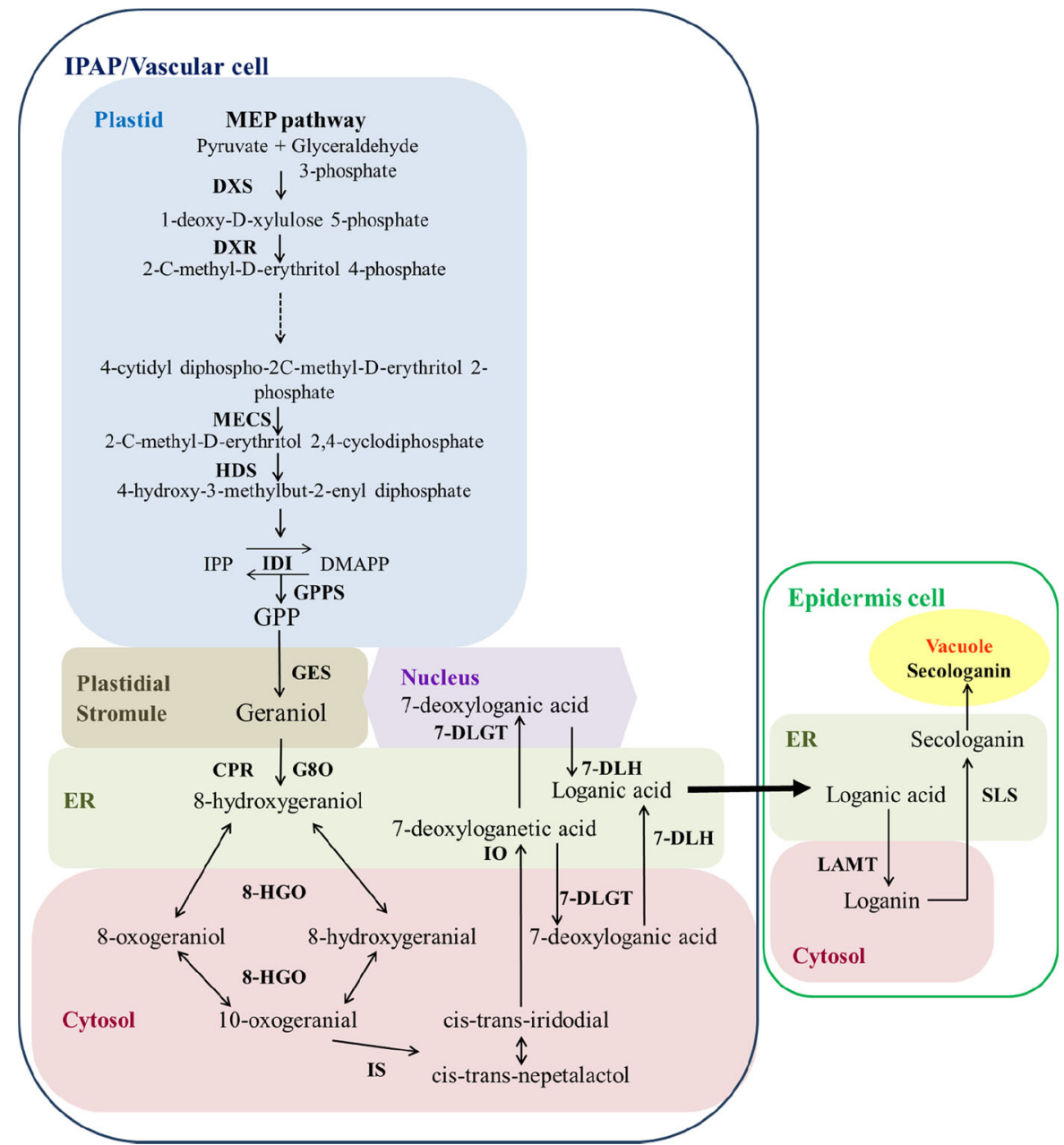

a certain extent in MIA biosynthesis, whereas CrGPPS is a homomeric enzyme forming GPP.

The secoiridoid pathway starts with the formation of geraniol and ends with the production of secologanin (Fig. 2). Until recently only three steps and their enzyme and genes in the pathway were well established. One is the cytochrome P450 enzyme geraniol 10-hydroxylase $(\mathrm{G} 10 \mathrm{H})$, also known as geraniol 8 -oxidase (G8O, the name we will use here), responsible for the hydroxylation of geraniol into 8-hydroxygeraniol (Collu et al. 2001; Meijer et al. 1993b), which also requires a cytochrome P450 reductase (CPR) for its functioning (Madyastha and Coscia 1979; Meijer et al. 1993a). The other two, are the last steps in the iridoid pathway, the enzymes loganic acid methyltransferase (LAMT) catalyzing methylation of loganic acid to form loganin (Murata et al. 2008) and secologanin synthase (SLS), belonging to the cytochrome P450 (CYP) family, catalyzing the conversion of loganin to secologanin (Luijendijk et al. 1998; Irmler et al. 2000). Recently, the enzymes geraniol synthase (GES) (Simkin et al. 2012), a member of the terpene synthase family, and iridoid synthase (IS), a cyclase recruited from short-chain oxidoreductase (Geu-Flores et al. 2012), were cloned and their function in $C$. roseus was confirmed. The purified recombinant GES protein catalyzes the conversion of GPP into geraniol with a $\mathrm{Km}$ value of 58.51 M for GPP. The enzyme IS functions for the cyclization of 8-oxogeranial into iridodial, which probably couples an initial $\mathrm{NAD}(\mathrm{P}) \mathrm{H}$-dependent reduction step via a Diels-Alder cycloaddition or a Michael addition (Geu-Flores et al. 2012). The enzymes for the remaining four steps were recently discovered and their encoding genes cloned (Asada et al. 2013; Miettinen et al. 2014; Salim et al. 2013). 
The enzyme after G8O, 8-hydroxygeraniol oxidoreductase (8-HGO) is able to oxidize the hydroxy group to an aldehyde in the substrates 8-hydroxygeraniol, 8-hydroxygeranial and 8-oxogeraniol in the presence of NAD+ to yield 8-hydroxygeranial, 8-oxogeraniol and 8-oxogeranial. The CYP enzyme iridoid oxidase (IO) catalyzes the conversion of iridodial into 7-deoxyloganetic acid, which is glucosylated by the enzyme 7-deoxyloganetic acid glucosyltransferase (7DLGT) forming 7-deoxyloganic acid using UDPglucose as the sugar donor. Then the 3rd to last step in secologanin biosynthesis is catalyzed by 7-deoxyloganic acid hydroxylase (7-DLH), also known as 7-deoxyloganic acid 7-hydroxylase (CrDL7H), which belongs to the same P450 subfamily as SLS, yielding loganic acid (Miettinen et al. 2014; Salim et al. 2013). With these findings the secoiridoid pathway has now been fully characterized (Fig. 2). The elucidation clearly shows the evolution in biosynthesis elucidation. The first steps were identified by purification of the enzymes, sequencing part of the amino acid sequence and based on that probes where constructed for picking up the genes. This required knowledge of the intermediates of every single step in a pathway, and the availability of these compounds as substrate for the enzyme assays. The lack of knowledge of the iridoid pathway was thus the major bottleneck in the past years. The recently developed fast sequencing methods opened new possibilities. For example, transcriptomic sequence data were obtained from $C$. roseus plants and cell cultures under different conditions. By comparing transcriptome, proteome and metabolome data from all these conditions, candidate genes could be selected and subsequently the encoded enzymes were overexpressed and tested for their putative activity, this approach was successful in the EU project Smartcell that aimed at elucidation of the total secoiridoid pathway (Dong et al. 2013; Miettinen et al. 2014). Another approach is to silence the selected genes and through the analysis of the intermediates found in the plant the function can be deduced. This strategy was applied by de Luca et al. who used a medicinal plants gene sequence database as basis (Asada et al. 2013; Salim et al. 2013).

Besides the elucidation of the MIA pathway, its cellular and subcellular localization was also investigated. The internal phloem associated parenchyma (IPAP) cells present in the periphery of stem pith or intraxylary on the upper part of the vascular bundles in leaves are the primary locations for the expression of genes in the MEP pathway (like DXR, DXS, MECS, $H D S, I D I$ ) (Mahroug et al. 2007; Burlat et al. 2004). RNA in situ hybridization showed that transcripts of GES, IS, 8-HGO, IO, 7-DLGT and 7-DLH along with $\mathrm{G} 8 \mathrm{O}$ are localized in the IPAPs like the genes involved in MEP pathway (Asada et al. 2013; Miettinen et al. 2014; Geu-Flores et al. 2012; Simkin et al. 2012). That means that these cells produce loganic acid which is transported to e.g. the epidermis cell, where the loganic acid is methylated by the enzyme LAMT. The epidermis harbors also the maximum expression of the secologanin synthase (SLS) gene product the CYP72A1 catalyzing the last oxidation step in the iridoid pathway leading to secologanin (Irmler et al. 2000; St-Pierre et al. 1999; Yamamoto et al. 2000). The carborundum abrassion (CA) approach led to the generation of the $C$. roseus leaf epidermome-enriched cDNA library containing the last two genes of the secologanin pathway ( $L A M T, S L S$ ), the tryptamin pathway $(T D C)$ as well as of the first step in the MIA pathway (STR, SGD) C. roseus (Murata et al. 2008). Detailed analysis of this data set clearly revealed its abundance for genes like SLS and LAMT transcripts. From these findings it is concluded that both the MEP pathway and early steps of the secoiridoid pathways take place in IPAP cells while the last two steps of the secoiridoid pathway are localized in the epidermis. Loganic acid is the mobile intermediate transferred from IPAPs to epidermis, which indicates that its transport might be a key biosynthetic rate controling point for the fluxes in secologanin production.

Subcellular compartmentation of the MEP-secoiridoid pathway was also sorted out. The MEP pathway occurs in plastids (Roytrakul and Verpoorte 2007). Besides, the presence of HDS was also evidenced in long stroma-filled thylakoid-free extensions budding from plastids (Guirimand et al. 2009). Expression of green fluorescent protein (GFP) fusions revealed that IDI is targeted to plastids, mitochondria and peroxisomes in C. roseus cells (Guirimand et al. 2012). GFP localization indicated that CrGPPS.SSu is plastidial whereas CrGPPS is mitochondrial (Rai et al. 2013). Transient transformation of $C$. roseus cells with a yellow fluorescent protein-fusion construct revealed that GES is localized in plastid stroma and stromules (Simkin et al. 2012). G8O has now definitely shown to be localized in the endoplasmic reticulum (ER) (Guirimand et al. 2009). IS was shown to be 
exclusively localized in the cytosol by using fluorescent protein fusions as well as bimolecular fluorescence complementation assays (Geu-Flores et al. 2012). Using GFP fusions in C. roseus cells together with mCherry markers, IO and 7-DLH were revealed to be ER-associated, whereas 8-HGO and 7-DLGT are soluble proteins present in both cytosol and nucleus (Miettinen et al. 2014). LAMT forms homodimers in the cytosol, whereas SLS is anchored in the ER via an $\mathrm{N}$-terminal helix (Guirimand et al. 2011a). This fits the model of loganic acid being the transported intermediate that after uptake in the epidermis cells is methylated in the cytosol and subsequently further oxidized in the ER to yield secologanin. All these studies suggest a potential channel to export the MEP pathway product GPP from plastids, via stromules, to ER- and cytosol-anchored enzymes for iridoid biosynthesis (Fig. 2).

The monoindole alkaloid biosynthesis and its localization

The biosynthesis of monoindole alkaloids starts from the central intermediate strictosidine, which is formed by a condensation reaction of tryptamine and secologanin catalyzed by strictosidine synthase (STR), from where the diversion of metabolic fluxes starts towards different MIA biosynthetic pathways (De Waal et al. 1995; Fig. 3). In C. roseus, STR has at least 7 isoforms which are likely to be the products of posttranslational modifications, most likely through glycosylation as was shown for Cinchona STR (Stevens et al. 1993), since STR is encoded by a single gene (Mcknight et al. 1990; Pasquali et al. 1999). Strictosidine undergoes a deglucosylation reaction by strictosidine- $\beta$-D-glucosidase (SGD) enzyme to yield an unstable aglycon, cathenamine, which is a quite reactive carbinolamine and depending on the environment it can occur in different forms (Barleben et al. 2007; Geerlings et al. 2000; Lounasmaa and Hanhinen 1998; Stöckigt et al. 1977). Cathenamine is the branching point for three routes to different types of MIAs: (1) reduction to form ajmalicine by the enzyme cathenamine reductase (CR), which is further oxidized to serpentine by peroxidase in the vacuoles; (2) conversion of the iminium form of cathenamine into tetrahydroalstonine with cofactor NADPH by tetrahydroalstonine synthase (THAS), which finally is oxidized into alstonine; (3) reversible conversion to 4,21-dehydrogeissoschizine, which can be routed

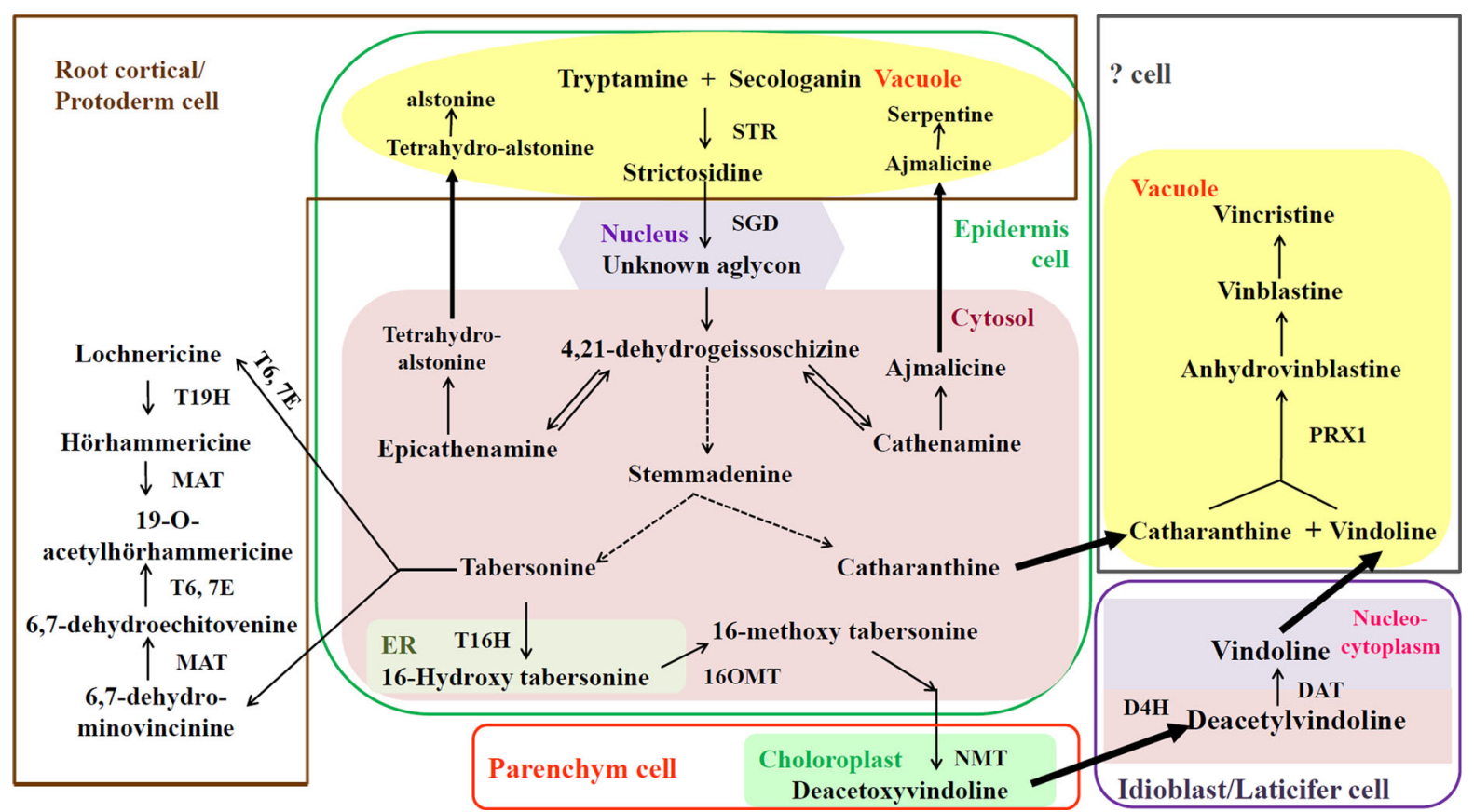

Fig. 3 Monoindole and bisindole alkaloids pathway and their compartmentation at intercellular and subcellular level. Solid arrows represent one-step reactions; broken arrows represent multiple or uncharacterized reactions. "? cell”: unclear cellular localization 
towards the formation of catharanthine and tabersonine/vindoline via stemmadenine. Neither enzymes nor genes involved in the catharanthine biosynthetic pathway have been identified so far (El-Sayed and Verpoorte 2007), but a unique transporter for catharanthine was reported recently (see below, Yu and De Luca 2013). On the other hand, the vindoline biosynthesis pathway beginning with tabersonine is quite well established. There are totally 6 enzyme-catalyzed steps in this pathway: hydroxylation of tabersonine by tabersonine 16-hydroxylase $(\mathrm{T} 16 \mathrm{H}) ; O$-methylation of 16-hydroxytabersonine by $O$-methyltransferase (16OMT); hydration of the 2,3-double bond of 16-methoxytabersonine by an unidentified hydroxylase; N(1)-methylation of 16-methoxy-2,3-dihydro-3-hydroxytabersonine by $\mathrm{N}$-methyltransferase (NMT); hydroxylation at position 4 of desacetoxyvindoline by desacetoxyvindoline-4-hydroxylase (D4H); and the last step the 4- $O$-acetylation of deacetylvindoline by deacetylvindoline-4- $O$-acetyltransferase (DAT) to form vindoline. Except the yet unknown hydroxylase, for the other five enzymes the genes have been cloned and characterized. Moreover, the first vindoline biosynthetic enzyme is reported to display two isoforms encoded by distinct genes characterized to date in C. roseus, CYP71D12 (T16H1) and CYP71D351 (T16H2). While T16H1 expression is restricted to flowers and undifferentiated cells, the $\mathrm{T} 16 \mathrm{H} 2$ expression profile is similar to the other vindoline biosynthetic genes reaching a maximum in young leaves (Besseau et al. 2013).

In roots, there are two alternative routes from tabersonine that instead of vindoline biosynthesis leads to other type of alkaloids (Verma et al. 2012). One is the conversion into lochnericine followed by the formation of hörhammericine, which is finally converted into $19-O$-acetylhörhammericine by minovincinine-19-hydroxy- $O$-acetyltransferase (MAT). The other route starts with the formation of 6,7dehydrominovincinine but the final product is also 19- $O$-acetylhörhammericine (Fig. 3).

In leaves, stems and flower buds, the epidermis cells harbor the STR mRNAs and idioblast and laticifer cells embedded in the palisade tissue of the leaves represent the exclusive location of $D 4 H$ and $D A T$ mRNAs transcripts (St-Pierre et al. 1999; Fig. 3). The $T 16 H 2$ and SGD mRNAs were mainly found in the epidermis (Murata and De Luca 2005; Murata et al. 2008; Besseau et al. 2013). High 16-OMT activity was detected in both abaxial and adaxial epidermal cell extracts, whereas the NMT activity is the highest in the whole leaf extract supporting its localization in the chloroplast thylakoids (Murata and De Luca 2005; Murata et al. 2008).

In the underground tissues, STR and MAT transcripts are localized in protoderm and cortical cells around the root apical meristem (Laflamme et al. 2001; Moreno-Valenzuela et al. 2003). The STR-GFP signal appeared in the vacuole in transient transformation experiments (Guirimand et al. 2010b). The vacuolar targeting of STR is achieved via an ER-toGolgi-to-vacuole route. SGD is targeted to the nucleus using a bipartite NLS and tends to multimerize in this cellular compartment (Guirimand et al. 2010a). For the vindoline pathway, subcellular localization is fairly well understood. T16H is anchored to ER as a monomer (St-Pierre et al. 1999), whereas 16OMT is found to homodimerize in the cytoplasm to facilitate the uptake of the $\mathrm{T} 16 \mathrm{H}$ conversion product. NMT is present in the thylakoid membrane of the chloroplasts (De Luca and Cutler 1987; Dethier and De Luca 1993), and D4H and DAT that were largely believed to operate in the cytosol of idioblast and laticifer cells have now been shown to operate as monomers and reside in the nucleocytoplasmic compartment because of their passive diffusion to nuclei due to their small protein size (Guirimand et al. 2011b).

The bisindole alkaloids and their localization

The first step of the bisindole alkaloid biosynthesis is the coupling of vindoline and catharanthine to produce anhydrovinblastine catalyzed by $\alpha-3^{\prime}, 4^{\prime}$-anhydrovinbastine synthase (AVLBS) (Fig. 3), which is commonly known as CrPRX1 belonging to the class III basic peroxidases (Costa et al. 2008). Anhydrovinblastine or its iminium ion is converted into vinblastine, which is finally converted into vincristine (Verpoorte et al. 1997). Peroxidases are a family of isoenzymes found in all higher plants and are known to be involved in a broad range of physiological processes. In C. roseus, more novel peroxidase genes are cloned and characterized, such as CrPRX, CrPRX3 and CrPRX4 (Kumar et al. 2007, 2011). However, there is still limited knowledge about the enzymes and genes involved in the last two steps. The subcellular localization researches reported that CrPRXI is 
localized to vacuoles (Costa et al. 2008), but $C r P R X$ is apoplastic in nature (Kumar et al. 2007).

\section{Metabolic engineering of the MIA pathway in Catharanthus roseus}

Metabolic engineering is an approach to modify metabolic pathways and metabolites production via gene transfer technology. With the increasing knowledge of the MIA pathway and its biosynthetic genes, metabolic engineering is widely performed on MIA biosynthesis in C. roseus to boost the yields of targeted MIAs and their analogs.

Natural-compound-targeted engineering strategies

Different types of genes cloned from the MIA pathway have been overexpressed in the cells, hairy roots and plants of $C$. roseus, which showed different effects on MIA biosynthesis as well as at the level of the full metabolome (Table 2).

Table 2 Overexpression of genes involved in MIA biosynthesis in the cell cultures, hairy roots and plants of Catharanthus roseus

\begin{tabular}{|c|c|c|c|}
\hline Varities & Genes & Metabolites significantly affected in levels & References \\
\hline \multirow[t]{18}{*}{ Hairy roots } & GmMYBZ2 & Catharanthine & Zhou et al. (2011) \\
\hline & $H M G R$ & Campesterol, serpentine, ajmalicine, catharanthine & Ayora-talavera et al. (2002) \\
\hline & $A S \alpha$ & Tryptophan, tryptamine, lochnericine & Hughes et al. (2004a) \\
\hline & $T D C$ & Serpentine & Hughes et al. (2004b) \\
\hline & $A S \alpha+T D C$ & Tryptamine & \\
\hline & $A S \alpha$ & Tryptamine & Hong et al. (2006) \\
\hline & $A S \alpha+A S \beta$ & Tryptophan, tryptamine & \\
\hline & $\begin{array}{l}A S \alpha \\
\quad+A S \beta+T D C\end{array}$ & Tryptamine & \\
\hline & $A S \alpha \beta$ & Naringin, catechin, salicylic acid & Chung et al. (2007) \\
\hline & $D A T$ & Hörhammericine & Magnotta et al. (2007) \\
\hline & $O R C A 2$ & Catharanthine, vindoline & Liu et al. (2011) \\
\hline & $G 8 O(G 10 H)$ & Catharanthine & Wang et al. (2010) \\
\hline & $G 8 O+O R C A 3$ & Catharanthine & \\
\hline & $D X S$ & $\begin{array}{l}\text { Ajmalicine, serpentine, lochnericine, tabersonine, } \\
\text { hörhammericine }\end{array}$ & Peebles et al. (2011) \\
\hline & $G 80$ & - & \\
\hline & $A s \alpha$ & $\begin{array}{l}\text { Tryptophan, tryptamine, lochnericine, tabersonine, } \\
\text { hörhammericine }\end{array}$ & \\
\hline & $D X S+G 8 O$ & Ajmalicine, tabersonine, lochnericine, hörhammericine & \\
\hline & $D X S+A s \alpha$ & $\begin{array}{l}\text { tryptamine, tabersonine, lochnericine, hörhammericine, } \\
\text { tryptophan }\end{array}$ & \\
\hline \multirow{7}{*}{$\begin{array}{l}\text { Cell } \\
\text { cultures }\end{array}$} & ORCA3 & Serpentine, ajmalicine, tabersonine, hörhammericine & Peebles et al. (2009) \\
\hline & $T D C$ & Tryptamine & $\begin{array}{l}\text { Canel et al. (1998), Whitmer et al. } \\
\text { (2002b) }\end{array}$ \\
\hline & $S T R$ & $\begin{array}{l}\text { Strictosidine, ajmalicine, catharanthine, serpentine, } \\
\text { tabersonine }\end{array}$ & $\begin{array}{l}\text { Canel et al. (1998), Whitmer et al. } \\
\text { (2002a) }\end{array}$ \\
\hline & $P R X 1$ & Ajmalicine, serpentine, $\mathrm{H}_{2} \mathrm{O}_{2}$ & Jaggi et al. (2011) \\
\hline & $O R C A 3$ & Tryptophan, tryptamine & van der Fits and Memelink (2000) \\
\hline & СYР76B6 & 10-hydroxy geraniol & Collu et al. (2001) \\
\hline & $C j M D R 1$ & Ajmalicine, tetrahydroalstonine & Pomahacová et al. (2009) \\
\hline \multirow[t]{3}{*}{ Plant } & $D A T$ & Vindoline & Wang et al. (2012) \\
\hline & $O R C A 3$ & Vindoline, catharanthine & Pan et al. (2012) \\
\hline & $O R C A 3+G 8 O$ & Strictosidine, vindoline, catharanthine & \\
\hline
\end{tabular}




\section{Structural genes}

The expression of a more tryptophan inhibition resistant Arabidopsis $A S \alpha$ enzyme coupled with a glucocorticoid-inducible promoter in $C$. roseus hairy roots dramatically increased tryptophan and tryptamine yields but not of MIAs, except lochnericine, after induction with $3 \mu \mathrm{M}$ dexamethasone (Hughes et al. 2004a). Transgenic hairy roots expressing both $A S \alpha$ and $A S \beta$ subunits produced more tryptamine and showed a greater resistance to feedback inhibition of AS activity by tryptophan than those only expressing $A S \alpha$ (Hong et al. 2006). When fed with the terpenoid precursors 1-deoxy-D-xylulose, loganin, and secologanin respectively, hairy roots overexpressing $A S \alpha$ or $A s \beta$ could increase the levels of hörhammericine, catharanthine, ajmalicine, lochneriricine and tabersonine (Peebles et al. 2006). As a side effect, the metabolic flux into the flavonoid pathway was also transiently increased when the $A S$ overexpressing hairy roots were induced by $0.2 \mu \mathrm{M}$ dexamethasone, which caused increases of catechin and naringin in hairy roots (Chung et al. 2007). This might be due to the induction of the phenylalanine/tyrosine pathway by tryptophan (Verpoorte and Alfermann 2000). TDC overexpression in $C$. roseus transgenic calli results in increased tryptamine levels but not in increased MIA production (Goddijn et al. 1995), neither in C. roseus cell cultures (Whitmer et al. 2002b). On the contrary, no increase of tryptamine but a $129 \%$ increase of serpentine was noted on induction of $3 \mu \mathrm{M}$ dexamethasone in hairy roots overexpressing TDC (Hughes et al. 2004b). Expressing TDC from C. roseus in cell cultures or plants of Nicotiana tabacum resulted in the formation of tryptamine up to $10 \mu \mathrm{g} \mathrm{g}^{-1} \mathrm{FW}$ and 18 to $66 \mu \mathrm{g} \mathrm{g}^{-1}$ FW respectively (Hallard et al. 1997). When co-overexpressing $A S$ and $T D C$ in hairy roots, an enhanced ability to produce tryptamine was observed, but only a transiently increased accumulation of tabersonine and lochnericine among all measured alkaloids (Hong et al. 2006; Hughes et al. 2004b). To study the effect of introducing MIA alkaloid biosynthetic genes in a plant normally only producing secologanin, Hallard et al. (Hallard 2000) introduced both the TDC and STR into Weigelia hairy roots. Compared to normal roots no more secologanin could be observed, whereas tryptamine, ajmalicine and serpentine could be detected in the hairy roots. This confirmed the presence of a glucosidase able to hydrolyze strictosidine. Though the alkaloids levels were very low, it shows that MIAs can also be made in non-alkaloid producing plants. That means alternative crops for making MIA. In that context also the production of strictosidine was achieved in yeast cells in which STR and SGD are overexpressed and which are fed with secologanin and tryptamine (Geerlings et al. 2001). The cells could produce $3 \mathrm{~g} \mathrm{l}^{-1}$ of strictosidine in 3 days, many times more than ever achieved in plant cell cultures. As STR was mainly excreted to the medium, whereas SGD was in the cells, grinding the whole culture resulted in the production of cathenamine. The transgenic yeast cells could be grown on the juice pressed out of the berries of Symphoricarpus albus rich in sugar and secologanin from which strictosidine was made after feeding tryptamine (Geerlings et al. 2001).

In the MEP pathway, the genes encoding $D X S, D X R$, 2-C-methyl-Derythritol-2,4-cyclodiphosphate synthase (MECS), hydroxymethylbutenyl-4-diphosphate $(H D S)$ and IDI1 have been cloned and characterized from C. roseus (Chahed et al. 2000; Veau et al. 2000; Guirimand et al. 2012). DXS overexpression resulted in a significant increase in ajmalicine, serpentine and lochnericine but a significant decrease in tabersonine and hörhammericine in $C$. roseus hairy roots. In fact, overexpression of $D X S$ and $D X R$ has been found to increase terpenoid production in several plants. For example, $D X S$ overexpression enhances the production of various isoprenoids in Arabidopsis (Estévez et al. 2001), and DXR overexpression increases essential oil yield in peppermint, carotenoid accumulation in ripening tomatoes (Mahmoud and Croteau 2001; Rodríguez-Concepción et al. 2001), and various isoprenoids in tobacco leaves (Hasunuma et al. 2008). There is no information about the use of MECS and IDII in metabolic engineering on MIA production.

At the edge of primary and secondary metabolism, G8O as gatekeeper could be a carbon flux controlling step for the iridoid pathway. The encoding gene was overexpressed in the hairy roots of $C$. roseus, which resulted in a higher accumulation of catharanthine (0.063-0.107\% of dry weight) than in the wild-type lines (0.019 and $0.029 \%)$ (Wang et al. 2010). When co-overexpressing DXS and $G 80$, the hairy roots showed a significant increase in ajmalicine by $16 \%$, lochnericine by $31 \%$ and tabersonine by $13 \%$ (Peebles et al. 2011). Considering their location with respect to the IPP/DMAPP branching point for 
terpenoid classes, it is conceivable that the overexpression of one downstream structural gene alone is unable to effect the channeling of the flux at this upstream branch point while the overexpression together with an upstream gene of the IPP/DMAPP branch point may affect the carbon fluxes by a push and pull effect toward the MIA iridoid precursor. An increased production of IPP/DMAPP and $G 8 O$ overexpression may increase the flux in the monoterpenoid branch and from there into MIA. DXS and $A S \alpha$ cooverexpression displayed a significant increase in hörhammericine by $30 \%$, lochnericine by $27 \%$ and tabersonine by $34 \%$ in hairy roots (Peebles et al. 2011). Recent discoveries of IDI1, GPPS, GES and iridoid synthase encoding genes provide new possibilities for the regulation and improvement of MIA production (Dong et al. 2013; Fischer et al. 2013).

Cultures of STR transgenic cells consistently showed ten-fold higher STR activity than wild-type cultures, which favored the biosynthetic flow through the pathway. Two such lines accumulated over $200 \mathrm{mg}^{-1}$ of strictosidine and strictosidine-derived MIAs, including ajmalicine, catharanthine, serpentine, and tabersonine, while maintaining wild-type levels of TDC activity (Canel et al. 1998). Whitmer et al. (2002a, b) showed that in C. roseus cell lines overexpressing TDC or STR have an overcapacity of indole alkaloid biosynthesis enzyme activities, as feeding of loganin resulted in a large increase of alkaloid production whereas the combination of loganin and tryptamine feeding even further increased the level of alkaloids. Apparently the iridoid pathway is the most limiting step, but when that limitation is overcome the tryptophan pathway becomes limiting. Overcoming one limiting step immediately shows what the next limiting step is. A single structural gene overexpression will thus always have only a limited effect on the overall flux in a pathway. On the other hand it shows that probably many biosynthetic steps are already present, and only the enzymatic machinery has to be started up by increasing the amount of the limiting substrate by feeding or genetic modification. The elucidation of the full iridoid pathway as described above is thus a major breakthrough opening new possibilities to explore for increasing MIA production.

The key gene DAT for the vindoline biosynthesis was introduced into $C$. roseus plants by Agrobacterium tumefaciens, which resulted in an increase of vindoline level in the leaves (Wang et al. 2012). However, overexpression of DAT in hairy roots altered their MIA profile and accumulated more hörhammericine compared to control lines (Magnotta et al. 2007). Comparative analysis revealed that MIA pathway genes have elevated expression levels in $C r P R X$ overexpression transgenic hairy roots, whereas they had a significant reduction in their transcript level in CrPRX-RNAi transgenic hairy roots (Jaggi et al. 2011). Alkaloid analysis showed higher levels of ajmalicine and serpentine in these peroxidase overexpressing cell lines. All these transgenic lines produced higher amounts of $\mathrm{H}_{2} \mathrm{O}_{2}$ (Jaggi et al. 2011). The oxidative burst or $\mathrm{H}_{2} \mathrm{O}_{2}$ production is closely related to indole alkaloid production (Zhao et al. 2001). In leaves of $C$. roseus, PRX together with phenolic compounds were suggested to represent an important sink of excess $\mathrm{H}_{2} \mathrm{O}_{2}$, diffusing from the chloroplast under high light exposure (Ferreres et al. 2011). These results indicate a role of the $C r P R X$ gene in the regulation of MIA pathway and other metabolic pathways, thus affecting the production of specific alkaloids. In order to study the role of CrPRX and CrPRX1 in plants, these two peroxidases were expressed in Nicotiana tabacum (Kumar et al. 2012). The transformed plants exhibited increased peroxidase activity. Increased oxidative stress tolerance was also observed in transgenics when treated with $\mathrm{H}_{2} \mathrm{O}_{2}$ under strong light conditions. However, under salt and dehydration stress, the seed germination of $\operatorname{CrPRX}$ transformed plants and wild-type plants was clearly suppressed, whereas $C r P R X 1$ transgenic lines showed improved germination. $C r P R X$-transformed lines exhibited better cold tolerance than CrPRX1-transformed lines. These results indicate that vacuolar peroxidases (e.g. CrPRX) play an important role in salt and dehydration stress, while cell wall-targeted peroxidases (e.g. CrPRX1) render cold stress tolerance (Kumar et al. 2012).

\section{Transporter genes}

Since MIA biosynthesis involves at least four different cell types and in each of them at least five different subcellular compartments, the trafficking of pathway intermediates from one to another compartment requires an efficient transport system. Previous research also suggested that transport is one of the 
potential factors in regulation of MIA biosynthesis. However, the knowledge about MIA membrane transport mechanisms is still very limited.

Transport has basically two aspects, a physicochemical and a biochemical one. In cells and in an organism diffusion will always take place. Concentration gradients make molecules to diffuse in a liquid phase. Moreover molecules will equilibrate between the aqueous phase and lipid phase (membrane). Mass transfer factors determine the rate of the uptake in a lipophilic membrane from water and the release again to water, i.e. they affect the transport rate through a membrane. That allows calculations of the rate of diffusion of compounds between cells and cellular compartments. The complexity of this system is further increased by the $\mathrm{pH}$, making that acids and bases at different $\mathrm{pH}$ have different solubility in the liquid phases. For example, an alkaloid in acidic conditions is poorly soluble in a lipid phase, but at basic conditions it is better lipid soluble. So at a high ratio of protonated to non-protonated alkaloids, which is at acidic conditions, transport will be slow through a membrane, at higher $\mathrm{pH}$ it will be the opposite. Modeling uptake in $C$. roseus vacuoles using these physical-chemical processes resulted in an ion-trap model for alkaloid uptake in vacuoles that fitted reasonably well the experimental results using isolated vacuoles. The lower $\mathrm{pH}$ in the vacuole than in the cytosol causes preferred accumulation of alkaloids in the vacuole if compared with the cytosol as the uptake rate on the more basic cytosolic site of the membrane is faster than on the more acidic vacuolar side. This physicochemical process requires ATP for maintaining the low vacuolar $\mathrm{pH}$, so depletion of ATP will inhibit uptake, similar as in case of $\mathrm{ABC}$ transporters (Blom et al. 1991). On the other hand Deus-Neumann and Zenk (1984) reported uptake kinetics for active transport for some indole alkaloids. Ajmalicine, catharanthine and vindoline showed different rates, and all were ATP dependent. From this it was hypothesized that the vacuolar transport occurred via selective transporter proteins. Roytrakul (2004) reported a detailed study on the uptake of several $C$. roseus alkaloids and secologanin in isolated vacuoles. By adding inhibitors of the various classes of transport proteins, for each individual compound quite a different and complex picture came out. For each single compound different transporter seems to be involved (Roytrakul and Verpoorte 2007).
The uptake into the vacuole is thus dependent on a combination of factors, first of all there is the bidirectional diffusion driven transport. On top of that there are multi drug resistant associated proteins (MRP, inhibited by glibenclamide) and ATP Binding Cassette (ABC, inhibited by ortho-vanadate) type of transporters involved in uptake. Whereas multidrug resistant (MDR) (P-glycoproteins, inhibited by cyclosporine A and verapamil) and MDR coupled with proton symport, are responsible for extrusion. To further complicate the transport system, glutathione was found to cis-activate the MRP transport of ajmalicine into the vacuole (Roytrakul 2004; Roytrakul and Verpoorte 2007). Considering the multicompartment system involved in the MIA biosynthesis, it is clear that with the already very complex transport system into vacuoles, the model for a single-cell or multi-cell system is impossible to describe. The need for sufficient energy and co-factors in the different compartments add further to this complexity. In an attempt to calculate the rate of transport between cells by using the various available data on uptake of compounds and a number of assumptions based on observations from other plants, it became clear that at least diffusion alone would result in a biosynthetic rate more or less of what is found in the plant (Supandi et al. 2009, unpublished results). That means that the selective transport might play a role in accumulating compounds in certain cells and in some of the specific biosynthetic steps, e.g. by accumulating certain compounds in a vacuole, where they are oxidized to yield serpentine or dimeric alkaloids. In case of serpentine, this anhydronium compound is much more polar than ajmalicine from which it is formed by oxidation, thus becomes trapped into the vacuole. The fact that tobacco vacuoles excrete strictosidine, whereas $C$. roseus vacuoles store it (Hallard et al. 1997) shows at least that every plant species will have different transport systems with different selectivity. Considering that the MIAs are confined to certain cell types may also in part be due to specific transport systems in the cellular membranes. That means that introduction of a novel pathway in a plant maybe hampered by lack of transport of intermediates.

The example of CjMDR1, an ABC transporter gene specific for berberine transport originally isolated from Coptis japonica, shows the problems one may encounter in genetically modifying transport. This 
gene was expressed in C. roseus cell cultures (Pomahacová et al. 2009). The endogenous alkaloids ajmalicine and tetrahydroalstonine were accumulated significantly more in $C$. roseus cells expressing CjMDRl in comparison with control lines after feeding these alkaloids, but transport of other alkaloids was not affected, and even no effect at all on berberine transport into the cells was observed.

A unique catharanthine $\mathrm{ABC}$-transporter (CrTPT2) belonging to the pleiotropic drug resistance (PDR) family has been cloned and functionally characterized. It is expressed predominantly in the epidermis of young leaves (Yu and De Luca 2013). Further analysis suggested that CrTPT2 may be specific to MIAproducing plant species, where it mediates secretion of alkaloids to the leaf surface. CrTPT2 gene expression is induced under the treatment with catharanthine, and its silencing redistributes catharanthine into the leave, causing an increase of dimeric alkaloids levels in the leaves.

Recently strong support for active MIAs uptake by C. roseus mesophyll vacuoles through a specific $\mathrm{H}(+)$ antiport system was reported (Carqueijeiro et al. 2013). The vacuolar transport mechanism of the main MIAs accumulated in $C$. roseus leaves, vindoline, catharanthine, and $\alpha-3^{\prime}, 4^{\prime}$-anhydrovinblastine, was characterized using a tonoplast vesicle system. Vindoline uptake was ATP dependent, and this transport activity was strongly inhibited by $\mathrm{NH}_{4}{ }^{+}$and carbonyl cyanide m-chlorophenyl hydrazine and was insensitive to the ATP-binding cassette (ABC) transporter inhibitor vanadate. Spectrofluorimetric assays with a $\mathrm{pH}$-sensitive fluorescent probe showed that vindoline and other MIAs indeed were able to dissipate an $\mathrm{H}^{+}$ preestablished gradient across the tonoplast by either vacuolar $\mathrm{H}^{+}$-ATPase or vacuolar $\mathrm{H}^{+}$-diphosphatase. Though it was claimed that this system would be responsible for the MIA transport instead of an iontrap mechanism or ABC transporters, it seems unlikely, as at least physicochemical based transport will always occur and the various previous reports found alkaloid specificity for the uptake into the vacuole.

\section{Transcription factors}

Transcription factors (TFs) are sequence-specificDNA-binding proteins that interact with the promoter regions of target genes and modulate the rate of
mRNA synthesis by RNA polymerase II (Gantet and Memelink 2002). They usually control the expression of more than one gene vital for normal development and functional physiology in plants. Several TFs have been found to be involved in the regulation of secondary metabolism. In C. roseus, MIA biosynthesis is related with plant defense and controlled by a number of signals including developmental cues, light, and biotic and abiotic stress. Regulation of MIA biosynthetic genes is coordinated by several types of TFs (Fig. 4).

The best-known TFs regulating MIA biosynthesis are the jasmonates-responsive ORCAs (octadecanoidresponsive Catharanthus AP2-domain proteins) from the plant-specific AP2/ERF (APETALA2/ethyleneresponsive factor) family, i.e. ORCA2 and ORCA3, for which the regulation mechanism of the MIA biosynthetic genes in $C$. roseus is well established. ORCAs expression is induced by jasmonates (Van der Fits and Memelink 2001), which is a major and essential signaling pathway to induce MIA biosynthesis. Jasmonates are first converted to the bioactive jasmonate isoleucine derivative (JA-Ile). Perception of JA-Ile by CrCOl1 causes the degradation of the CrJAZ proteins, derepressing the bHLH TF, CrMYC2 protein. CrMYC2 then activates the expression of ORCAs, which in its turn activate the expression of MIA biosynthetic genes through binding to the JERE (jasmonate and elicitor-responsive element) in the promoter of targeted genes (Menke et al. 1999; Van der Fits and Memelink 2000; Zhang et al. 2011). Another bHLH TF, CrMYC1, binds specifically to the G-box of STR gene promoter in yeast (Chatel et al. 2003). Ectopic expression of ORCA3 in cell cultures of $C$. roseus increased the expression of the MIA biosynthetic genes TDC, STR, $C P R$ and $D 4 H$, as well as two genes encoding primary metabolic enzymes ( $A S$ and $D X S$ ) (Van der Fits and Memelink 2000). This indicates that ORCA3 is a central regulator of MIA biosynthesis and positively regulates the biosynthesis of MIAs and their precursors. Nevertheless, ORCA3 does not regulate the expression of $G 8 O$ and DAT. Overexpression of ORCA3 caused an increase of ajmalicine and serpentine but a decrease in tabersonine, lochnericine, and hörhammericine in hairy roots (Peebles et al. 2009). When ORCA3 combined with $G 8 O$ were overexpressed in hairy roots, alkaloid accumulation level analyses showed that all transgenic clones accumulated more catharanthine, with the 
Fig. 4 Gene regulation scheme of the reported transcription factors involved in the MIA pathways. Dashed lines represent interactions that may be direct or indirect. Solid lines indicate potentially direct interactions. Lines with arrows represent transactivation, and lines with bars represent transrepression. $G A$ gibberellic acid, $E T$ ethylene. Enzymes in black represent to be regulated by reported transcription factors, enzymes in grey represent that their regulation has not been reported and remained unclear

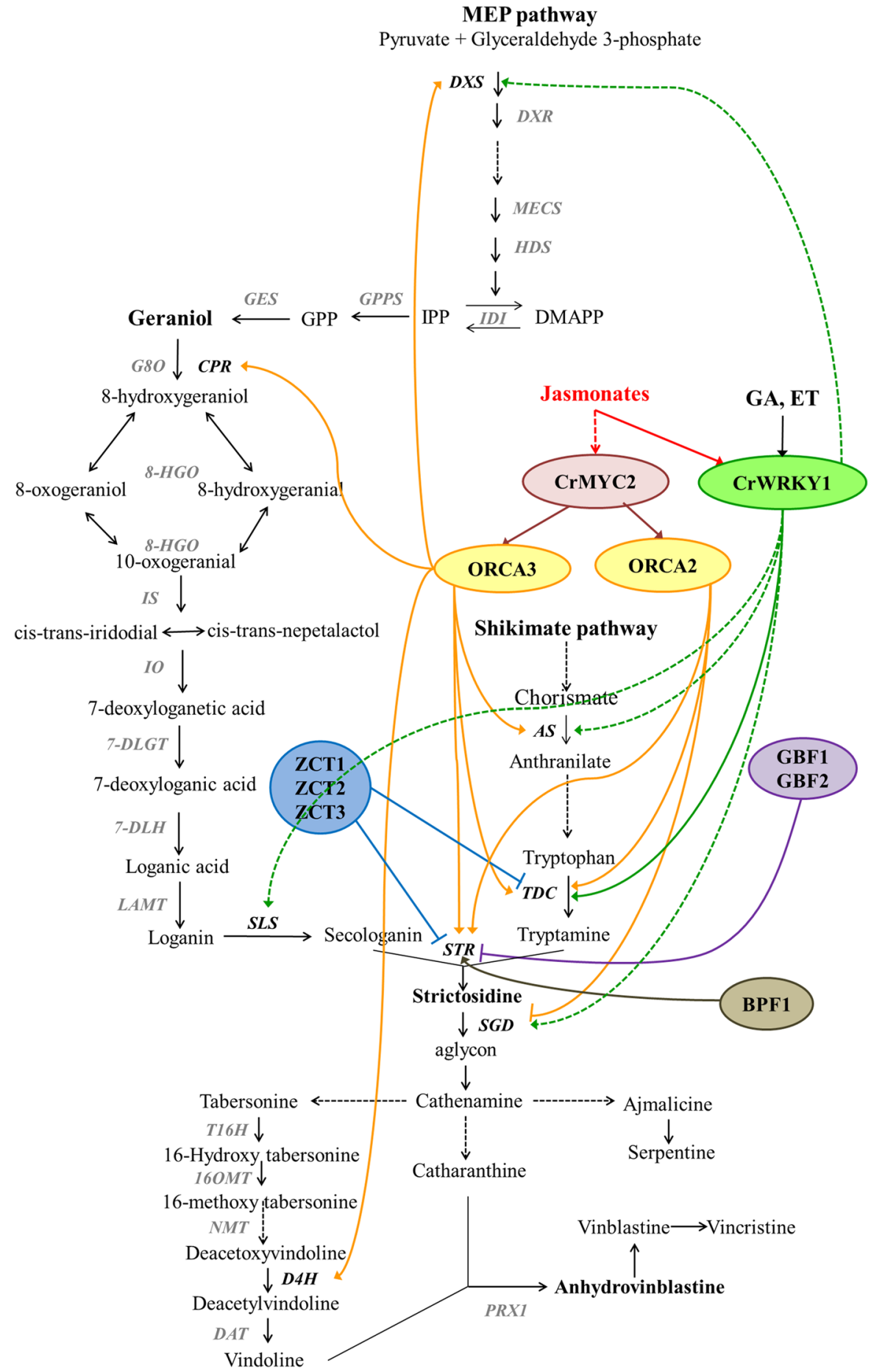

highest accumulation level 6.5-fold more than that of the non-expression clone (Wang et al. 2010). ORCA2 from $C$. roseus was demonstrated to regulate the expressions of $S T R, T D C$ and $S G D$ gene, but has no effect on the CYP related reductase $(C P R)$, which is regulated by ORCA 3 (Li et al. 2013; Menke et al. 1999). Transgenic hairy root cultures overexpressing ORCA2 showed an average content of catharanthine 
that was increased up to 2.03 in comparison to the control lines, respectively. However, vinblastine could not be detected in the transgenic and control hairy root cultures by HPLC (Liu et al. 2011).

The zinc finger-binding proteins ZCT1, ZCT2, and ZCT3 (members of the transcription factor IIIA-type zinc finger family) were found to bind to the promoters of STR and TDC. This interaction repressed the activity of STR and TDC. The binding of the ZCTs to the $S T R$ promoter has been suggested to counteract the activation of STR by ORCA2 or ORCA3 (Pauw et al. 2004).

Using an enhancer domain of the STR promoter as bait in a yeast one-hybrid screen resulted in the isolation of $C r B P F 1$, a periwinkle homolog of the MYB-like transcription-factor BPF1 from parsley (Van der Fits et al. 2000). CrBPFl expression is induced by elicitors but not jasmonates, which indicates that elicitors induce $S T R$ expression in periwinkle cells via jasmonic-acid-dependent and -independent pathways.

Sequence analysis of the $S T R$ and $T D C$ promoters shows that they contain a G-box or G-box-like binding site. Two G-box-binding factors, CrGBF1 and CrGBF2, were subsequently identified in C. roseus and shown to repress the transcription of STR by binding to the G-box sequence (Sibéril et al. 2001).

A C. roseus WRKY transcription factor, CrWRKY1, is preferentially expressed in roots and induced by the phytohormones jasmonate, gibberellic acid and ethylene (Suttipantaa et al. 2011). Overexpression of CrWRKY1 in C. roseus hairy roots upregulated several key MIA pathway genes, especially $T D C$, as well as transcriptional repressors ZCT1, ZCT2 and ZCT3. However, $C r W R K Y 1$ overexpression repressed the transcriptional activators, ORCA2, ORCA3 and CrMYC2. Overexpression of a dominant repressive form of CrWRKY1, created by fusing the SRDXrepressor domain to CrWRKY1, resulted in downregulation of $T D C$ and $Z C T s$ but up-regulation of ORCA3 and CrMYC2. CrWRKY1 binds to the W-box elements of the TDC promoter in the electrophoretic mobility shift, yeast one-hybrid and $C$. roseus protoplast assays. Up-regulation of TDC increased TDC activity, tryptamine concentration and resistance to 4-methyl tryptophan inhibition of $C r W R K Y 1$ hairy roots. Compared to control roots, CrWRKYI hairy roots accumulated variable but significant increase of serpentine (from $131.5 \pm 23.7$ to $291.5 \pm 73.2 \mu \mathrm{g} \mathrm{g}^{-1}$ ) and ajmalicine (from $12.8 \pm 1.4$ to $15.4 \pm 1.6 \mu \mathrm{g} \mathrm{g}^{-1}$ ). The preferential expression of $C r W R K Y 1$ in roots and its interaction with transcription factors including ORCA3, CrMYC2 and ZCTs may play a key role in determining the root-specific accumulation of serpentine and ajmalicine in $C$. roseus plants.

The root-specific MADS-box transcription factor Agamous-like 12 (Agl12) from Arabidopsis thaliana was expressed on the differentiation of suspension cells from $C$. roseus (Montiel et al. 2007). The expression of Agl12 is sufficient to promote an organization of suspension cells into globular parenchyma-like aggregates but is insufficient by itself to induce complete morphological root differentiation. Agl12 expression selectively increases the expression of genes encoding enzymes involved in the early biosynthetic steps of the terpenoid precursor of the alkaloids. The transgenic cell lines expressing Agl12 produced significant amounts of ajmalicine, which indicates that TFs involved in tissue or organ differentiation may constitute new metabolic engineering tools to produce specific valuable MIAs. Murata and De Luca (2005) reported that ORCA3 and an AP2/ERF type of transcription factors were expressed in all four cell types (epidermis, IPAP, laticifers and idioblast cells).

Although different types of TFs have been reported to interact with the genes in the MIA pathway, regulation of the key enzyme genes involved in its branches still remains unclear and need to be figured out, such as the iridoid pathway, vindoline pathway and bisindole alkaloids pathway.

Besides TFs involved in TIA biosynthesis, signal components were found to have a role in accumulation of TIAs in response to abiotic stress. CrMPK3, a noval mitogen activated protein kinase (MAPK), was isolated and found activated by wounding, UV treatment and MeJA in C. roseus leaves (Raina et al. 2012). Transient overexpression of CrMPK3 in C. roseus leaf showed enhanced expression of key TIA biosynthetic genes and also accumulation of specific TIAs (Raina et al. 2012).

Unnatural-compound-targeted engineering strategies

Approaches to generate new-to-nature compounds from plant-based pathways are also developed on $C$. roseus, which modifies the structure of a natural product to improve the biological activity of the 
compound. Replacement of an endogenous starting material with an unnatural compound is a strategy that has been broadly applied in prokaryotic biosynthetic pathways (O'Connor 2012). Now genetic manipulation is performed on the MIA pathway combined with precursor-directed biosynthesis and engineered enzymes to produce various unnatural products in $C$. roseus.

RNA mediated suppression of tryptamine biosynthesis in $C$. roseus hairy root culture eliminates the production of MIAs derived from tryptamine and secologanin. But when an unnatural tryptamine analog, 5-fluorotryptamine 1a, was fed to both wildtype and silenced cultures, a variety of novel fluorinated alkaloids, such as fluoro-ajmalicine, fluorotabersonine and fluoro-serpentine, were produced and not contaminated with the natural alkaloid counterparts in silenced lines (Runguphan et al. 2009). The flux of the unnatural substrate could be enhanced to the downstream alkaloids through some branches of the pathway when the natural, endogenous substrate is limited or unavailable. Targeted silencing of substrate biosynthesis combined with precursors feeding programs a plant alkaloid pathway to more effectively produce desirable novel products, which opens new areas of combining synthesis and biosynthesis to increase chemodiversity.

A mutant strictosidine synthase gene with reengineered substrate specificity was transformed into $C$. roseus. The resulting transgenic plant cell culture produced a variety of unnatural alkaloids compounds when cocultured with simple, achiral, commercially available precursors that the reengineered enzyme was designed to accept (Runguphan and O'Connor 2009). This work demonstrates the power of engineering new structures of complex alkaloidal natural products in plant cultures.

Another example is to validate the function of the engineered flavin-dependent halogenase RebH. In vivo, the tryptamine-specific RebH mutant (Y455W) was transformed into the alkaloid-producing plant $C$. roseus, and the de novo production of the halogenated alkaloid 12-chloro-19, 20-dihydroakuammicine was observed. The resulting tissue cultures accumulated substantial levels of 7-chlorotryptophan while wild-type (WT) RebH has been integrated into periwinkle metabolism previously. By installing chlorine onto tryptamine, the RebH Y455W mutant circumvents the bottleneck that tryptophan decarboxylase accepts 7-chlorotryptophan at only $3 \%$ of the efficiency of the native substrate tryptophan. In comparison with cultures harboring RebH and WT RebF, tissue cultures containing mutant RebH Y455W and RebF also accumulate microgram per gram freshweight quantities of 12-chloro-19,20-dihydroakuammicine but, in contrast, do not accumulate 7-chlorotryptophan, demonstrating the selectivity and potential utility of this mutant in metabolic engineering applications (Glenn et al. 2011).

The development of approaches to generate newto-nature compounds from $C$. roseus MIA pathway will produce a number of MIA analogs which have improve or alter biological activity, and will further enhance our ability to hijack the downstream MIA pathways (O'Connor 2012).

\section{Transcriptomics and proteomics on Catharanthus roseus}

The genes, enzymes and intermediates of the MIA pathway and its precursor pathways were found and annotated one by one. However, there are still a lot of mysteries in the pathway for its complex temporal and spatial regulation. To unravel the regulatory mechanisms behind the MIA biosynthesis, exploitation of modern transcriptomic and proteomic approaches has been carried on to complement the efforts of enhancing the MIA yields and provide more information about the metabolic network in $C$. roseus.

Transcriptome sequencing and assembly provides a rapid and inexpensive approach to access gene sequences, expression abundances and expression patterns in any species, even those that lack a reference genome sequence (Góngora-Castillo and Buell 2013). In $C$. roseus, the early effort toward transcriptome analysis was to generate expressed sequence tags (ESTs) from the leaf and root tissues by two different approaches: (1) indirectly through separate cDNA libraries; and (2) directly through a strategically designed suppression subtractive hybridization (SSH) using the leaf and root cDNAs as tester and driver, respectively (Shukla et al. 2006). A total of 155 ESTs were subjected to homology-based classification. Moreover, 16 novel ESTs from the direct approach and 12 from the indirect approach were obtained and need to be further characterized to ascertain their prospective roles in MIA biosynthesis 
(Shukla et al. 2006). De Luca et al. generated two cDNA libraries from RNA isolated from the base part of young leaves and from root tips to select 9824 random clones for unidirectional sequencing, to yield 3327 related sequences and 1696 singletons by cluster analysis. Putative functions of 3663 clones were assigned, from 5023 non-redundant ESTs to establish a resource for transcriptome analysis and gene discovery in this medicinal plant (Murata et al. 2006).

However, the collection of ESTs is limited to a few hundred or thousand sequenced cDNAs. Next generation sequencing (NGS), as high throughput methods, with computational approaches were used to generate large-scale transcriptome sequence (reads), assemblies and expression profiles for $C$. roseus with a substantial number of the assembled transcripts being full or near-full lengh (Góngora-Castillo et al. 2012). A total of 86,725 transcripts and 32,607 unique transcripts or unigenes were identified (GóngoraCastillo et al. 2012). Of the 362 documented C. roseus nuclear-encoded peptide sequences in GenBank, 290 $(80.1 \%)$ were identified, all of which were full-length or near full-length with $>80 \%$ coverage. Then, CathaCyc, a detailed metabolic pathway database, was constructed from $C$. roseus RNA-Seq data sets, which contains 390 pathways with 1347 assigned enzymes and spans primary and secondary metabolism (Van Moerkercke et al. 2013). As a part of CathaCyc, a C. roseus RNA-seq atlas was created and holds all publicly available $C$. roseus expression data derived from RNA-Seq experiments. CathaCyc also links the enzymes annotated to the online genome annotation web interface ORCAE (Van Moerkercke et al. 2013). The database will facilitate to the further study and exploitation of the metabolism of C. roseus.

With the development of high throughput RNAseq, transcriptome assembly using short reads presents bioinformatics challenges to be minimized to improve data interpretation. Whole-transcriptome profiles using NGS were analyzed to investigate the mechanism of the leafy flower transition in C. roseus during PnWB infection (Liu et al. 2014). Using de novo assembly approach generated more than 60,000 contigs and annotated $34.2 \%$ of the contigs as putative genes through name-calling, open reading frame determination and gene ontology (GO) analyses. The expression levels of $87.8 \%$ genes were consistent with those in the microarray profiles, suggesting that using NGS can detect accurate gene expression levels.
The data revealed a change of gene expression levels in S4 PnWB-infected leafy flowers. The network analysis suggested that the expression levels of $>1000$ candidate genes were highly associated with CrSVP1/ 2 and CrFT expression, which might be crucial in the leafy flower transition (Liu et al. 2014). In another case, deep transcriptome sequencing generated about 343 million reads from different tissues (leaf, flower and root) of C. roseus using the Illumina platform (Verma et al. 2014). Optimization of de novo assembly involving a two-step process resulted in a total of 59,220 unique transcripts with an average length of $1284 \mathrm{bp}$. In total, $65 \%$ of $C$. roseus transcripts showed homology with sequences available in various public repositories, while the remaining $35 \%$ unigenes may be considered as $C$. roseus specific. In silico analysis revealed presence of 11,620 genic simple sequence repeats (excluding mono-nucleotide repeats) and 1820 transcription factor encoding genes in $C$. roseus transcriptome (Verma et al. 2014).

In order to find novel proteins involved in the alkaloid biosynthesis of $C$. roseus, proteomic approach has been undertaken. Systematic analysis of the proteome was performed on $C$. roseus cell suspension culture A11, which accumulates strictosidine, ajmalicine and vindolinine, by two-dimensional polyacrylamide gel electrophoresis (Jacobs et al. 2005). 88 protein spots were selected for identification by MALDI-MS/MS, of which 58 were identified, including 12-ox-ophytodienoate reductase, TDC and two isoforms of STR (Jacobs et al. 2005). Besides key enzymes of the MIA biosynthetic pathway, there are active transporters required for transmembrane and intercellular translocation processes of MIA intermediates. However, the information of this kind proteins is quite limited. To further investigate the proteins involved in MIA synthesis and transport, the proteomes of two independent $C$. roseus cell lines with different MIA metabolism were compared by 2DDIGE, which revealed 358 proteins differing quantitatively by at least a twofold average ratio (Champagne et al. 2012). A total of 1663 proteins were identified. Among them, 63 enzymes are potentially involved in secondary metabolism, of which 22 are involved in MIA biosynthesis and 16 are predicted transporters putatively involved in secondary metabolite transport (Champagne et al. 2012).

Based on large-scale transcriptomic and proteomic database, a rapid strategy has been developed to 
discovery candidate genes of the key steps in TIA biosynthesis. For example, comparative heat map of some known MEP, iridoid and MIA pathway enzymes was made in C. roseus and visualized in Tree View to candidate gene products found in Catharanthus ovalis, Catharanthus longifolius, Amsonia hubrichtii, Vinca minor, Rauvolfia serpentina, Tabernaemontana elegans, Lonicera japonica, Cinchona ledgeriana and Camptotheca acuminata. protein amino (De Luca et al. 2014). The amino acid identities of genes from different species were imported into Cluster 3.0 (Michiel de Hoon, University of Tokyo; http:// bonsai.hgc.jp/_mdehoon/software/cluster/) and hierarchical clustering of species was done with uncentered correlation and centroid linkage to select genes. The selected candidate gene is then tested with a toolkit, including gene expressioin profiling, virus induced gene silencing, targeted metabolite profiling and functioinal identification of recombinant proteins, leading to functional gene discovery and pathway elucidation of TIA biosynthesis for further use in metabolic engineering (De Luca et al. 2014).

In conclusion, large-scale transcriptomic and proteomic study provides a rich and huge source of data for understanding plant-specialized metabolism. The information represents an important reference database for a more functional analysis of the MIA pathway and metabolic network in C. roseus.

\section{NMR-based metabolomics on Catharanthus roseus}

As MIA biosynthesis is such a complex system, multiple techniques are required to elucidate the pathways and its regulation thereof. In the postgenomic era, metabolomics is the latest tool for functional genomics (Sumner et al. 2003). Metabolomics is a fast growing powerful technology and is useful for phenotyping and diagnostic analyses of plants (Schauer and Fernie 2006). It is rapidly becoming a key tool in functional annotation of genes and in the comprehensive understanding of the cellular response to various biological conditions. Metabolomic approaches have recently been used to assess the natural variance in metabolite content between individual plants, an approach with great potential for the improvement of the compositional quality of crops. Metabolomics covers metabolic profiling, fingerprinting, footprinting and metabolic flux analysis. Metabolomics can be used to measure the effect of developmental stage, environment, daily and seasonal changes and stress on the plant metabolome. Also for characterization and identifying varieties, chemotaxonomy and quality control of plant products (e.g. food, medicinal plants) metabolomics is a powerful tool. All metabolomics information can eventually be used in functional genomics, as for example shown for the elucidation of the iridoid pathway in C. roseus (Miettinen et al. 2014). Metabolomics in combination with transcriptomics thus became a major tool of functional genomics. Moreover, metabolomics is becoming an important tool in the quality control of food and medicinal plants, as well as a diagnostic tool in health care.

Nuclear magnetic resonance spectroscopy (NMR) is a very powerful method that allows the simultaneous detection of diverse groups of secondary metabolites (flavonoids, alkaloids, terpenoids, etc.) besides the abundant primary metabolites (sugars, organic acids, amino acids, etc.; Kim et al. 2010). The nonselectiveness of NMR makes it an ideal tool for unbiased plant metabolomics studies. As signals are proportional to their molar concentration in an NMR spectrum, it is possible to make the direct comparison of concentrations of all compounds without the need for calibration curves of each individual compound. Moreover, the time of analysis is short and the sample preparation is simple and fast, enabling the analysis of large numbers of samples per hour, without the need for calibration curves for all single compounds for quantitation. These are major advantages if compared with the more sensitive methods as MS, LC-MS and GC-MS, which all suffer from lack of absolute quantitative data. It is thus a choice between the quality of data: a large number of metabolites with only relative quantitation for each compound, or smaller number of metabolites with full quantitation. However, one should keep in mind that in all present metabolomics methods, the visible metabolome is determined, or may be better to say limited, by the method of extraction! Only soluble compounds might be visible, and poorly soluble compounds will always be present up to saturation levels, thus not showing any variation above that level, even when large differences in accumulation may occur in the plant. As the first choice for metabolomics, NMR-spectroscopy has been applied to plant metabolomic studies of $C$. roseus in every aspect, i.e. identification of novel metabolites, elucidation of metabolic pathways, 
metabolic responses to stress, metabolic characterization and classification, and metabolic flux analysis. NMR is also a very useful technique for structure elucidation using various 2D NMR measurements without further fractionation of the extract. Thus dozens of primary and secondary metabolites were identified in cell cultures, hairy roots, and plants of $C$. roseus (Table 3 ).

Metabolic profiling/fingerprinting combined with multivariate data analysis

Metabolite profiling, fingerprinting and footprinting are commonly used as efficient methods in metabolomics studies. Profiling aims at quantitative analysis of sets of metabolites in a selected biochemical pathway or a specific class of compounds. Fingerprinting uses high throughput qualitative screening of the metabolic composition in an organism or tissue with the primary aim of sample comparison and discrimination analysis. Footprinting is the fingerprinting analysis of metabolites that are excreted by cells to the culture medium. Multivariate or pattern recognition techniques such as the well-described unsupervised principal component analysis (PCA) hierarchical cluster analysis (HCA), and supervised partial least squares-discriminant analysis (PLS-DA) are useful tools to analyze complex data sets (Sumner et al. 2003). PCA and PLS-DA are the widely applied regression methods used to reduce the multidimensionality of the metabolomics data. They provide an excellent platform to study, for example, the stress response in plants; quality control and authentication of medicinal plants (like Artemisia annua, Angelica acutiloba, and Panax notoginseng); classification of different plant species genotypes or ecotypes; identification of biomarkers for disease diagnosis; or identification of bioactive compounds in plants. The unsupervised methods show the maximum separation between all samples in two or three dimensions. If this does not result in any clear grouping of the samples, supervised methods can be applied to reveal possible characteristic differences between certain defined classes. Metabolite profiling combined with other tools is being used to rapidly narrow down candidate genes for functional discovery of their biochemical roles (De Luca et al. 2014). In fact metabolomics has become the tool for systems biology, studying the response of the whole system, rather than a reductionist approach in which only a few parameters are measured. At the same time there is also the question what is the metabolome of a plant, in fact it is the mixture of the metabolome of different tissues and even different cells. One may thus speak about a macrometabolome and a micrometabolome, and even nanometabolome if one regards the role of cellular compartments in the cellular metabolism. The above discussed localization of the MIA biosynthesis over different cells and cellular compartments shows that for a better understanding of the biosynthesis a single cell or at least single cell type metabolomics would be of great value. First experiments in that direction have already been made. The analysis of epidermis cells is a clear example (Murata and De Luca 2005; Murata et al. 2008).

To understand the stress response in C. roseus, a comprehensive metabolomic profiling of the leaves infected by 10 types of phytoplasmas was carried out using one-dimensional and two-dimensional NMR spectroscopy followed by PCA (Choi et al. 2004). The results showed that major discriminating factors to characterize the phytoplasma-infected $C$. roseus leaves from healthy ones were increases of metabolites related to the biosynthetic pathways of phenylpropanoids and MIAs, chlorogenic acid, loganic acid, secologanin, and vindoline. Furthermore, higher abundance of glycine, glucose, polyphenols, succinic acid, and sucrose were detected in the phytoplasma-infected leaves. The effect of salicylic acid (SA) on the metabolic profile of $C$. roseus cell cultures in a time course $(0,6,12,24,48$ and $72 \mathrm{~h}$ after treatment) was studied using ${ }^{1} \mathrm{H}-\mathrm{NMR}$ spectroscopy and PCA (Mustafa et al. 2009b). Adding $25 \mu \mathrm{mol}$ of sodium SA into $100 \mathrm{~mL}$ of 5 days-old cell cultures altered the metabolome compared with the nontreated cells. A dynamic change in amino acids, phenylpropanoids, and tryptamine was found in cells at $48 \mathrm{~h}$ after SA treatment. Additionally, 2,5-dihydroxybenzoic-5- $O$-glucoside was detected only in SAtreated cells (Mustafa et al. 2009b).

Based on the metabolite profiles using ${ }^{1} \mathrm{H}-\mathrm{NMR}, C$. roseus plants were able to genetically discriminate between eight cultivars (Kim et al. 2007). Hierarchical dendrograms based on the aromatic region of $C$. roseus NMR data was in general agreement with the genetic relationships determined by conventional DNA fingerprinting methods. According to the signal assignment of the ${ }^{1} \mathrm{H}-\mathrm{NMR}$ spectra, secologanin and polyphenols contributed most to the discrimination between cultivars (Kim et al. 2007). ${ }^{1} \mathrm{H}-\mathrm{NMR}$-based 


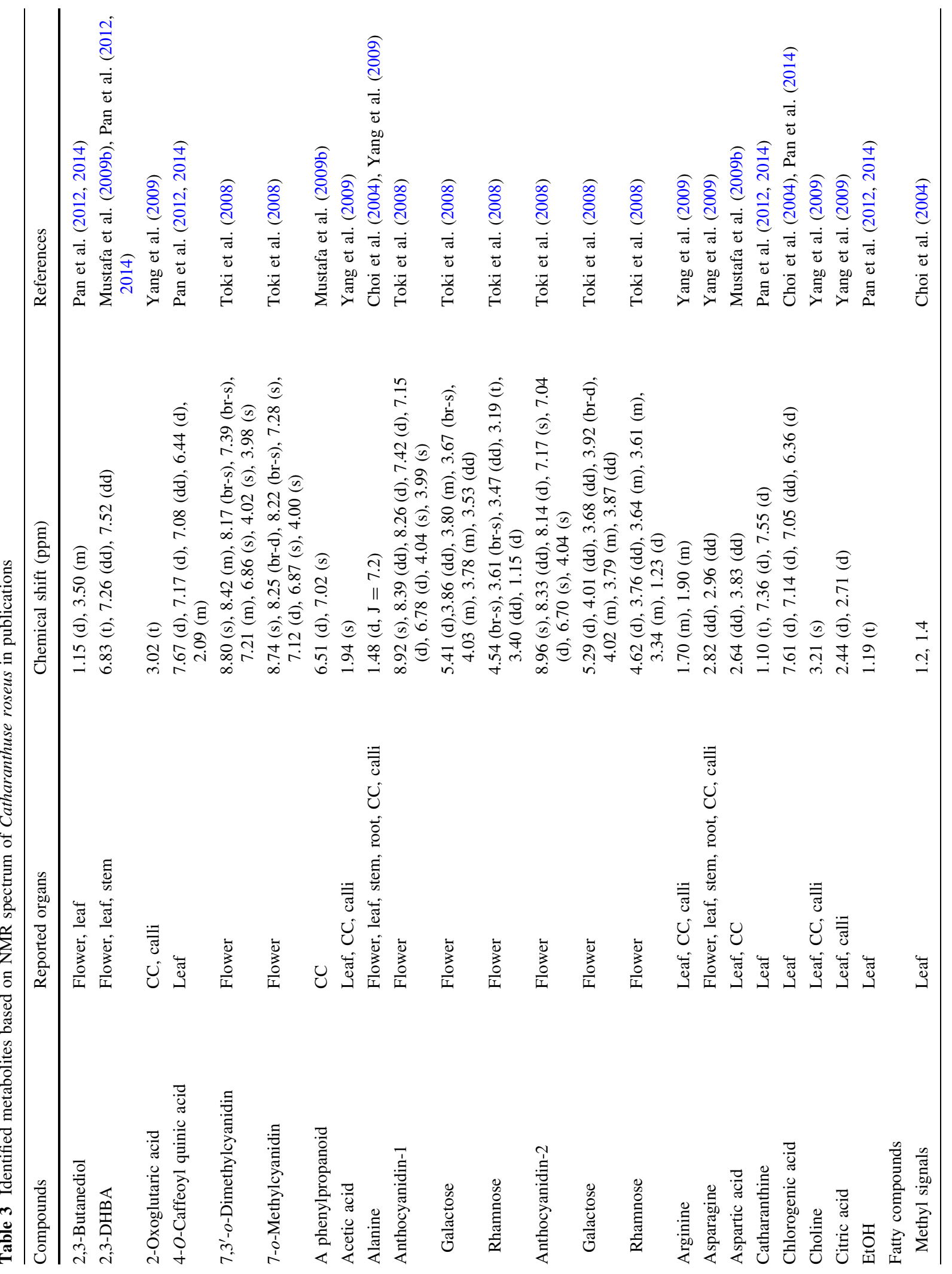




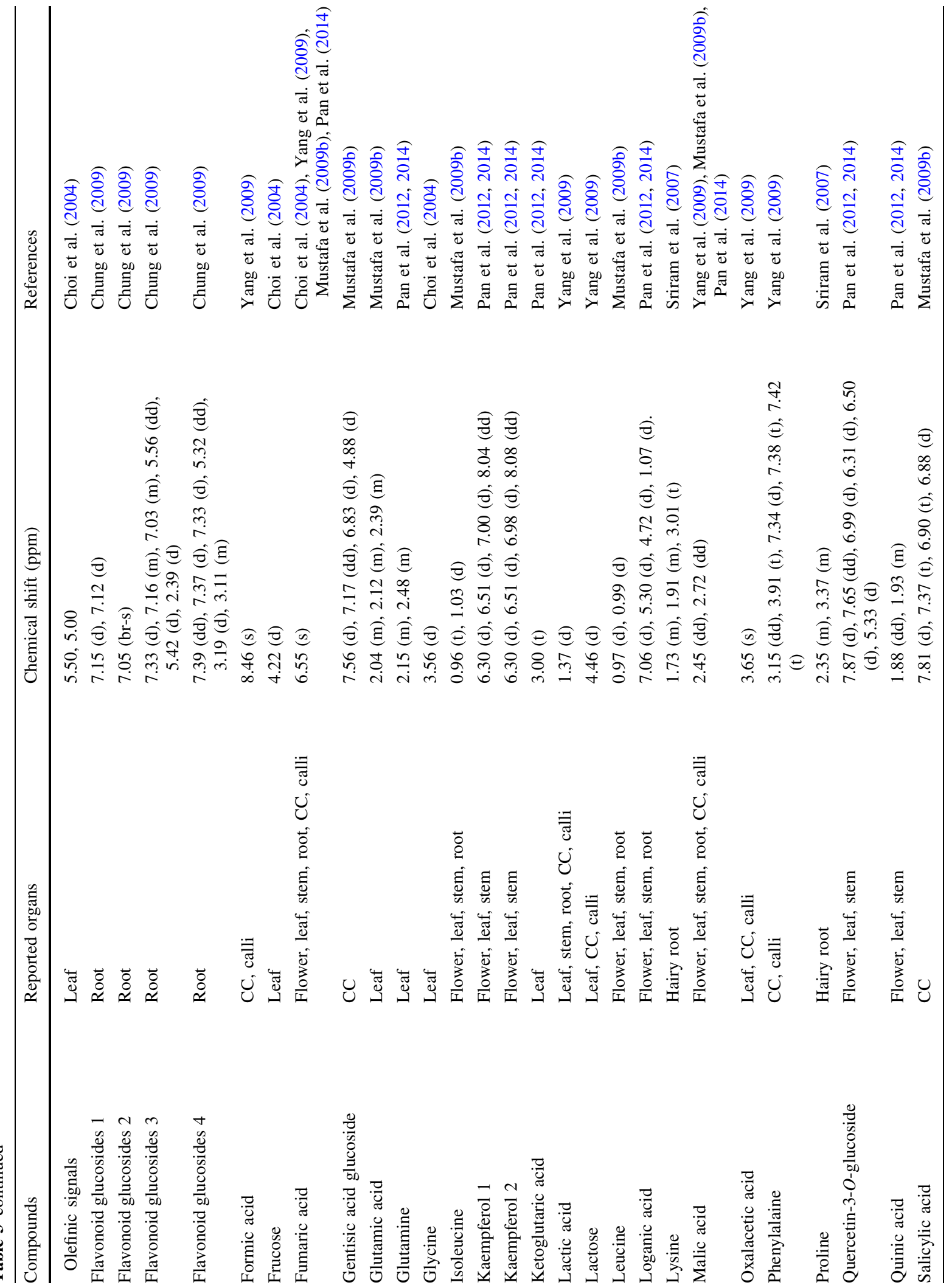




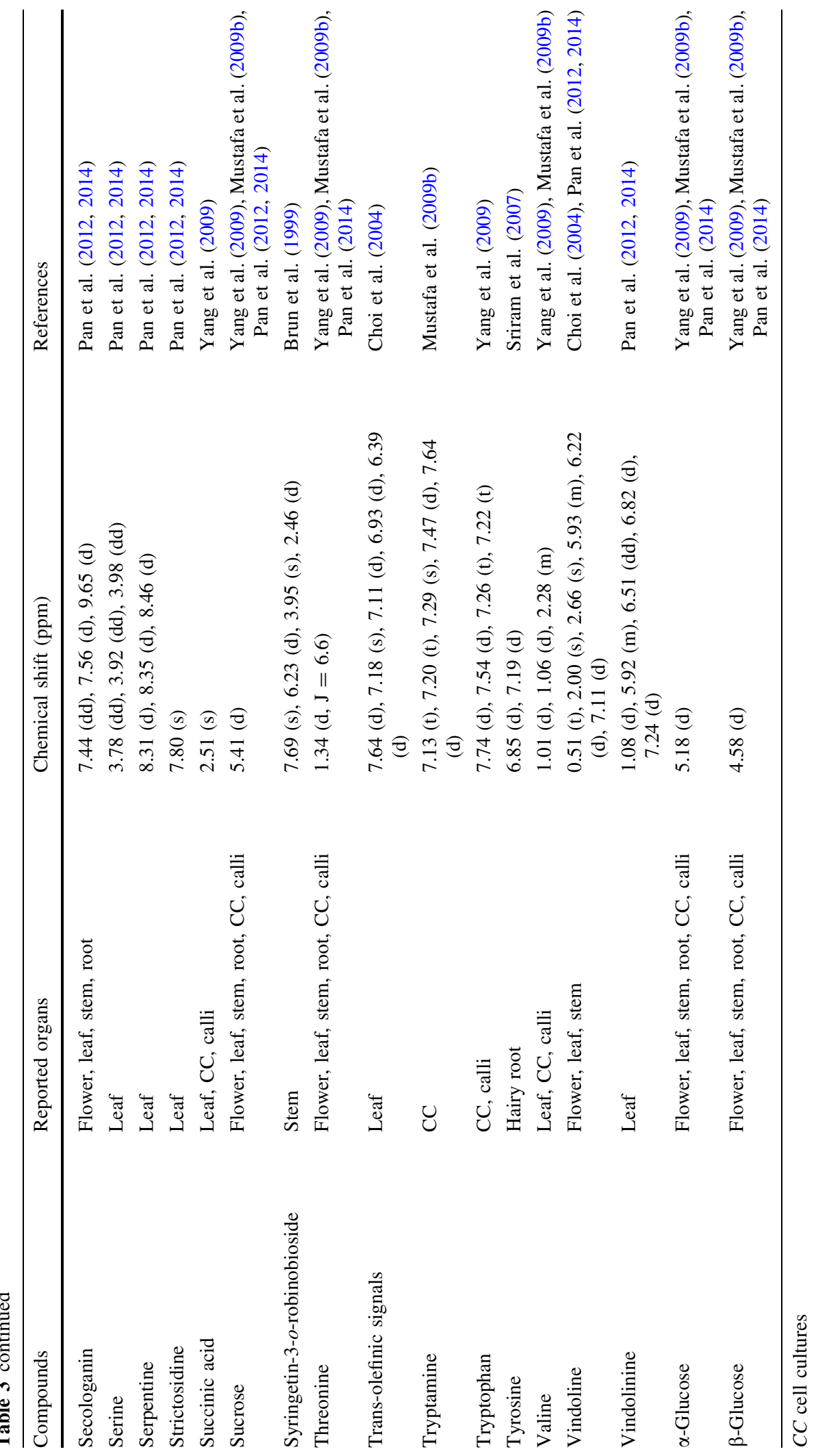


metabolomics analysis can differentiate inner and outer calli of $C$. roseus (Yang et al. 2009). The cells with different localization in the calli treated with different elicitors and relative locations could be separated in the PCA score plots. The levels of valine, threonine, alanine, asparagine, phenylalanine, tryptophan, choline, lactose, lactic acid, acetic acid, malic acid, succinic acid, citric acid, fumaric acid and formic acid were found higher in the inner callus than in the outer callus, whereas 2-oxoglutaric acid, oxalacetic acid, sucrose and glucose dominated in the outer callus. In another study, ${ }^{1} \mathrm{H}-\mathrm{NMR}$ and multivariate data analysis were used to characterize the metabolites and investigate the metabolic profiles of leaves, stems, roots and flowers of $C$. roseus with four flower colors (orange, pink, purple, and red; Pan et al. 2014). The results showed that flower color is characterized by a special pattern of metabolites such as anthocyanins, flavonoids, organic acids, and sugars. Not only the flowers but the leaves, stems, and roots also presented metabolic differences correlating with the flower color. Most importantly it seems possible to predict the flower color through profiling the metabolites in leaves, stems, or roots, which may be a helpful tool for plant breeding.

To better understand the effect of genetic engineering on plant metabolism, transgenic C. roseus plants overexpressing ORCA3 alone (OR lines), or cooverexpressing $\mathrm{G} 10 \mathrm{H}$ and ORCA3 (GO lines) were investigated by metabolomics (Pan et al. 2012). ${ }^{1} \mathrm{H}-$ NMR-based metabolomics confirmed the higher accumulation of monomeric indole alkaloids (strictosidine, vindoline, catharanthine and ajmalicine) in OR and GO lines. Moreover, multivariate data analysis of ${ }^{1} \mathrm{H}-\mathrm{NMR}$ spectra showed a clear separation between transgenic and control lines, which was determined by a change of amino acids, organic acids, sugars and phenylpropanoids levels in both OR and GO lines compared to the controls. The results indicate that enhancement of MIA biosynthesis by ORCA3 and G10H overexpression might also affect other metabolic pathways in the metabolism of $C$. roseus plants.

\section{Metabolic flux analysis based on ${ }^{13} \mathrm{C}$ labeling experiment}

One of the problems of metabolomics is that it is like a two-dimensional picture, it measures the amounts of compounds present at a certain time point, but it does not tell anything about the turnover. A major compound can be a stored product or part of a very active metabolic pathway, only measuring the dynamics of the system can give the answer that means measuring the flux through pathways, making a film, rather than a picture.

Metabolic flux analysis (MFA), the quantification of all intracellular fluxes in an organism, is thus an important cornerstone of metabolic engineering and systems biology. Each flux reflects the function of a specific pathway within the network. As all biological activity is related to metabolic activity, it is these fluxes that deliver the phenotype of an organism (Ratcliffe and Shachar-Hill 2005). Flux measurements complement transcriptomic, proteomic, and metabolomic technologies in defining phenotypes, and provide a useful complementary parameter for the system-wide characterization of metabolic networks. MFA on different phenotypes in plants can provide valuable information, which facilitates to select metabolic engineering targets, elucidate metabolic pathways, and construct metabolic models (Stephanopoulos and Stafford 2002). Metabolic flux analysis is usually carried out using ${ }^{13} \mathrm{C}-\mathrm{NMR}$ and ${ }^{1} \mathrm{H}_{-}{ }^{13} \mathrm{C}$ HSQC NMR analysis, GC-MS or LC-MS in experiments where ${ }^{13} \mathrm{C},{ }^{2} \mathrm{H}$ or ${ }^{15} \mathrm{~N}$ isotope-labeled compounds are added to the organisms studied. The chromatographic approaches do allow determining the overall percentage of labeling, but not like in NMR, the site of incorporation. The data are analysed and interpreted by mathematical models and software like ${ }^{13} \mathrm{C}-\mathrm{FLUX}^{\mathrm{TM}}$ and 4F (Ratcliffe and Shachar-Hill 2006). The ${ }^{13} \mathrm{C}$ isotope is widely used since it is not radio-active and NMR analysis allows determining the precise site of the label in a molecule. Natural abundance of ${ }^{13} \mathrm{C}$ is $1.1 \%$, so already a labeling of $1.1 \%$ will lead to a doubling of the percentage of the labeled carbons and consequently to a clear increase of the signal concerned. ${ }^{13} \mathrm{C}$ labeling experiments $\left({ }^{13} \mathrm{CLE}\right)$-based MFA have been applied to $C$. roseus for pathway elucidation, for finding crosslinks between pathways, and for flux quantification in the central carbon metabolism.

In C. roseus, mevalonate was first considered to be the exclusive precursor of isopentenyl diphosphate in the biosynthesis of secologanin. However, later research indicated that the MEP pathway might be the alternative pathway involved. A feeding experiment using $\left[1-{ }^{13} \mathrm{C}\right]$ glucose to $C$. roseus cell cultures followed by analysis of its incorporation into 
secologanin using ${ }^{13} \mathrm{C}$ NMR spectroscopy was performed. The data on the sites of incorporation of the ${ }^{13} \mathrm{C}$ label showed that the MEP pathway and not the mevalonate pathway was the major route for secologanin biosynthesis (Contin et al. 1998). The biosynthetic pathways of SA and 2,3-DHBA were studied through a similar feeding-NMR method. The data led to the conclusion that the isochorismate pathway is responsible for the biosynthesis of both compounds, presenting the first full chemical evidence for the isochorismate pathway for the biosynthesis of SA as an important signal molecule in plants (Mustafa et al. 2009a; Budi Muljono et al. 2002a, b).

In order to assess quantitatively the crosstalk between the MEP pathway and the mevalonate pathway, $\left[2-{ }^{13} \mathrm{C}_{1}\right]$ mevalonolactone or $\left[\mathrm{U}_{-}{ }^{13} \mathrm{C}_{6}\right]$ glucose were supplied to $C$. roseus cell cultures grown in light or dark (Schuhr et al. 2003). The incorporations of exogenous $\left[2-{ }^{13} \mathrm{C}_{1}\right]$ mevalonolactone were 48 and $7 \%$ into the DMAPP and IPP precursors of sitosterol and lutein, respectively. With $\left[\mathrm{U}_{-}{ }^{13} \mathrm{C}_{6}\right]$ glucose as precursor, at least $95 \%$ of sitosterol precursors were obtained from the mevalonate pathway, whereas phytol appeared to be biosynthesized via the deoxyxylulose phosphate pathway (approximately $60 \%$ ) as well via the mevalonate pathway (approximately $40 \%$ ).

Hairy roots of $C$. roseus, as a pharmaceutically significant plant compounds production system and an important metabolic engineering target, were used as a model system in the study of CLE-based MFA. [U- ${ }^{13} \mathrm{C}_{6}$ ] glucose was fed to the hairy roots of $C$. roseus to investigate its elemental and biomolecular composition, in which the abundances of lipids, lignin, cellulose, hemicellulose, starch, protein, proteinogenic amino acids, mineral ash, and moisture were quantified (Sriram et al. 2006). Moreover, 12 biomass synthetic fluxes were precisely calculated in the metabolic map of the plant system of $C$. roseus hairy roots. The results show the flux of the carbons from $\beta$ glucose consumed by the hairy roots into various products, which enables the design of metabolic engineering strategies to divert carbon to the economically attractive MIAs (Sriram et al. 2006). The application of "bondomers", which are isomers of a metabolite differing in the connectivity of their C-C bonds, was introduced to MFA study as a computationally alternative to the isotopomer concept in $C$. roseus (Sriram et al. 2007). Hairy roots were cultured on $\left(5 \%\right.$ w/w [U- $\left.{ }^{13} \mathrm{C} 6\right], 95 \%$ w/w naturally abundant $)$ sucrose. HSQC and COSY spectra of the hydrolyzed aqueous extract were acquired from the hairy roots. Analysis of these spectra yielded a data set of 116 bondomers of beta glucans and proteinogenic amino acids from the hairy roots. Fluxes were evaluated from the bondomer data by using comprehensive bondomer balancing, most of which were identified in a threecompartment model of central carbon metabolism with good precision. Pentose phosphate pathways were observed to occur in parallel in the cytosol and plastids with significantly different fluxes. The fluxes between phosphoenolpyruvate and oxaloacetate in the cytosol and between malate and pyruvate in the mitochondria were relatively high $(60.1 \pm 2.5 \mathrm{~mol}$ per $100 \mathrm{~mol}$ sucrose uptake, or $22.5 \pm 0.5 \mathrm{~mol}$ per 100 mol mitochondrial pyruvate dehydrogenase flux).

The development of a comprehensive flux analysis tool for the plant system of $C$. roseus is expected to be valuable in assessing the metabolic impact of genetic or environmental changes.

\section{Combination of omics tools for system biology}

Integration of multiple omics with various traits of plants is exercised to predict gene functions and characterize the complex interaction and coordination of plant metabolic network in biological processes from a system biological point of view (Fukushima et al. 2009). Combination of non-targeted approaches, such as transcriptomics and metabolomics, can reveal potential gene-to-metabolite networks (UrbanczykWochniak et al. 2003), filter out candidate genes for certain metabolic pathways (Okazaki et al. 2009), and suggest gene functions by overexpression (Lackman et al. 2011). The integration of omics approaches can help to reveal the organization of the whole system and thus to identify interesting targets for further studies. A comprehensive profiling analysis of $C$. roseus was performed by combining genome-wide transcript profiling of cDNA-amplified fragment-length polymorphism with metabolic profiling of elicited $C$. roseus cell cultures to yield a collection of known and previously undescribed transcript tags and metabolites associated with MIAs (Rischer et al. 2006). Previously undescribed gene-to-gene and gene-to-metabolite networks were drawn up by searching for correlations between the expression profiles of 417 gene tags and the accumulation profiles of 178 metabolite peaks. These networks revealed that the different branches of 
MIA biosynthesis and various other metabolic pathways are subject to different hormonal regulation. These networks also served to identify a select number of genes and metabolites likely to be involved in the MIA biosynthesis. So, the combination of multiple omics tools should contribute greatly to identification of key regulatory steps and characterization of the pathway interaction in various processes, aiming at elucidating the systemic coordination and communication among plant metabolic network.

\section{The future prospects}

With the rapid development of novel tools of modern plant molecular biology and chemistry, the knowledge of MIA biosynthesis and its regulation in C. roseus has greatly improved in the past decades. However, there are still quite a few unexplored parts of the pathway. More research is needed for discovering the missing structural genes, enzymes and intermediates of the pathway, as well as genes involved in the regulation of the pathway. This knowledge is needed to develop genetically modified plants, plant cells or microorganisms for the commercial production of the very valuable dimeric alkaloids. So far the genetic modification of the plant, plant cell cultures or microorganisms did not lead to the desired economically feasible production of MIA. In fact it seems that the pathway is more complex than just a series of enzyme catalyzed steps. Compartmentation of the pathway in the plant involving several different cell types and in these cells of all compartments is one of the complicating factors. This complexity makes it unlikely that a cell suspension culture will be able to perform all steps of the pathway. For a successful biosynthesis all the logistics must be in place, which means that every intermediate and all necessary cofactors and ATP required for the biochemical reactions are present on the right time, in the right quantity, on the right place.

Moreover, the MIA pathway does not exist independently in the total metabolic network of the plant but crosslinks and interacts with other branching pathways, which means it is part of a complex matrix, which raises the question how much of the total carbon flux in the plant can be channeled into MIA biosynthesis. To eventually solve all these problems a systems biology approach is required, which means that all omics will be needed to identify the missing links in the MIA biosynthetic pathway, and map the dynamics of the system. The availability of the full sequences from transcriptomics of many indole alkaloid producing plants under different conditions will be of great use to identify the structural genes of the pathways. The regulatory genes might be more difficult as in the different species the regulation of the biosynthesis can be different. Even in a single plant the regulation will be different between different MIA producing tissues, and between single cells dealing with different parts of the pathway. The single cell approach will thus be a major tool for unraveling pathways and its regulation and the physiological role of the alkaloids for the plant. Concerning the compartmentation and transport also the analysis of single cell metabolome will be important as it is likely that the different cell types involved will have different patterns of selective transporters for uptake and extrusion of intermediates in the different cellular compartments and for the whole cell itself. For example, in a leave there will probably be transporters for extrusion of loganic acid in the iridoid producing cells, whereas epidermis cells will have a transporter protein for the selective uptake of this compound. Non alkaloid producing cells may lack this transporter, or have a transporter that extrudes again this intermediate. Also the specific accumulation of the alkaloids in certain cells requires specific uptake and/or extrusion of alkaloids.

There is thus still a long and challenging way to go for complete understanding of the MIA biosynthesis. This knowledge will be of great value for our overall understanding of secondary metabolism in plants. Such research with no doubts will also generate knowledge that can lead to improved production of the dimeric alkaloids, similarly like the finding of the mechanism of the coupling of catharanthine and vindoline (Goodbody et al. 1988) has resulted in an industrial scale chemical production of the dimers from the readily available monomers! This made the monomers as the prime production target instead of the dimeric alkaloids.

Only genetic/molecular tools are not sufficient to figure out the landscape of MIA biosynthesis and regulation. Metabolomics, as a powerful technique to reveal changes in metabolic fluxes, is the ultimate level of post-genomic analysis and facilitates to get a deeper insight in the function of genes, pathways and single cells through a systems biology approach. 
Combination of metabolomics with other "omics" will speed up the elucidation of the MIA pathway and lead to breakthroughs in overcoming the bottlenecks in the production of MIAs in C. roseus.

Acknowledgments This work was funded by China national High-Tech "863" Program (Grant No. 2011AA100605) and Shanghai Key Discipline Cultivation and Construction Project (Horticulture).

\section{References}

Arigoni D, Sagner S, Latzel C et al (1997) Terpenoid biosynthesis from 1-deoxy-D-xylulose in higher plants by intramolecular skeletal rearrangement. Proc Natl Acad Sci USA 94:10600-10605

Asada K, Salim V, Masada-Atsumi S et al (2013) A 7-Deoxyloganetic acid glucosyltransferase contributes a key step in secologanin biosynthesis in Madagascar Periwinkle. Plant Cell Online 113:115154

Ayora-Talavera T, Chappell J, Lozoya-Gloria E et al (2002) Overexpression in Catharanthus roseus hairy roots of a truncated hamster 3-hydroxy-3-methylglutaryl-CoA reductase gene. Appl Biochem Biotech 97:135-145

Barleben L, Panjikar S, Ruppert M et al (2007) Molecular architecture of strictosidine glucosidase: the gateway to the biosynthesis of the monoterpenoid indole alkaloid family. Plant Cell 19:2886-2897

Besseau S, Kellner F, Lanoue A et al (2013) A Pair of Tabersonine 16-hydroxylases initiates the synthesis of vindoline in an organ-dependent manner in Catharanthus roseus. Plant Physiol 163:1792-1803

Blom T, Sierra M, Van Vliet T et al (1991) Uptake and accumulation of ajmalicine into isolated vacuoles of cultured cells of Catharanthus roseus (L.) G. Don. and its conversion into serpentine. Planta 183:170-177

Brun G, Dijoux MG, David B et al (1999) A new flavonol glycoside from Catharanthus roseus. Phytochemistry 50:167-169

Budi Muljono RA, Scheffer JJC, Verpoorte R (2002a) Isochorismate is an intermediate in 2,3-dihydroxybenzoic acid biosynthesis in Catharanthus roseus cell cultures. Plant Physiol Biochem 40:231-234

Budi Muljono RA, Talou JR, Scheffer JJC et al (2002b) Biosynthesis of 2,3-dihydroxybenzoic acid in transgenic Catharanthus roseus cell cultures overexpressing isochorismate synthase. Heterocycles 56:341-352

Burlat V, Oudin A, Courtois M et al (2004) Co-expression of three MEP pathway genes and geraniol 10-hydroxylase in internal phloem parenchyma of Catharanthus roseus implicates multicellular translocation of intermediates during the biosynthesis of monoterpene indole alkaloids and isoprenoid-derived primary metabolites. Plant J 38:131-141

Canel C, Lopes-Cardoso M, Whitmer S et al (1998) Effects of over-expression of strictosidine synthase and tryptophan decarboxylase on alkaloid production by cell cultures of Catharanthus roseus. Planta 205:414-419
Carqueijeiro I, Noronha H, Duarte P et al (2013) Vacuolar transport of the medicinal alkaloids from Catharanthus roseus is mediated by a proton driven antiport. Plant Physiol 162:1486-1496

Chahed K, Oudin A, Guivarc'h N et al (2000) 1-Deoxy-D-xylulose 5-phosphate synthase from periwinkle: cDNA identification and induced gene expression in terpenoid indole alkaloid-producing cells. Plant Physiol Biochem 38:559-566

Champagne A, Rischer H, Oksman-Caldentey KM et al (2012) In-depth proteome mining of cultured Catharanthus roseus cells identifies candidate proteins involved in the synthesis and transport of secondary metabolites. Proteomics 12:3536-3547

Chatel G, Montiel G, Pré M et al (2003) CrMYC1, a Catharanthus roseus elicitor- and jasmonate-responsive bHLH transcription factor that binds the G-box element of the strictosidine synthase gene promoter. J Exp Bot 54:2587-2588

Choi YH, Tapias EC, Kim HK et al (2004) Metabolic discrimination of Catharanthus roseus leaves infected by phytoplasma using ${ }^{1} \mathrm{H}-\mathrm{NMR}$ spectroscopy and multivariate data analysis. Plant Physiol 135:2398-2410

Chung IM, Hong SB, Peebles CAM et al (2007) Effect of the engineered indole pathway on accumulation of phenolic compounds in Catharanthus roseus hairy roots. Biotechnol Prog 23:327-332

Chung IM, Ahmad A, Ali M et al (2009) Flavonoid glucosides from the hairy roots of Catharanthus roseus. J Nat Prod 72:613-620

Collu G, Unver N, Peltenburg-Looman AM et al (2001) Geraniol 10-hydroxylase, a cytochrome P450 enzyme involved in terpenoid indole alkaloid biosynthesis. FEBS Lett 508:215-220

Contin A, van der Heijden R, Lefeber AWM et al (1998) The iridoid glucoside secologanin is derived from the novel triose phosphate/pyruvate pathway in a Catharanthus roseus cell culture. FEBS Lett 434:413-416

Costa MMR, Hilliou F, Duarte P et al (2008) Molecular cloning and characterization of a vacuolar class III peroxidase involved in the metabolism of anticancer alkaloids in Catharanthus roseus. Plant Physiol 146:403-417

Courdavault V, Papon N, Clastre M et al (2014) A look inside an alkaloid multisite plant: the Catharanthus logistics. Curr Opin Plant Biol 19:43-50

De Luca V, Cutler AJ (1987) Subcellular localization of enzymes involved in indole alkaloid biosynthesis in Catharanthus roseus. Plant Physiol 85:1099-1102

De Luca V, Salim V, Thamm A et al (2014) Making iridoids/ secoiridoids and monoterpenoid indole alkaloids: progress on pathway elucidation. Curr Opin Plant Biol 19:35-42

De Waal A, Meijer AH, Verpoorte R (1995) Strictosidine synthase from Catharanthus roseus: purification and characterization of multiple forms. Biochem J 306:571-580

Dethier M, De Luca V (1993) Partial purification of an $N$ methyltransferase involved in vindoline biosynthesis in Catharanthus roseus. Phytochemistry 32:673-678

Deus-Neumann B, Zenk M (1984) A highly selective alkaloid uptake system in vacuoles of higher plants. Planta 162:250-260 
Dong L, Miettinen K, Goedbloed M et al (2013) Characterization of two geraniol synthases from Valeriana officinalis and Lippia dulcis: similar activity but difference in subcellular localization. Metab Eng 20:198-211

Eisenreich W, Menhard B, Hylands PJ et al (1996) Studies on the biosynthesis of taxol: the taxane carbon skeleton is not of mevalonoid origin. Proc Natl Acad Sci USA 93:6431-6436

El-Sayed M, Verpoorte R (2007) Catharanthus terpenoid indole alkaloids: biosynthesis and regulation. Phytochem Rev 6:277-305

Estévez JM, Cantero A, Reindl A et al (2001) 1-deoxy-D-xylulose5-phosphate synthase, a limiting enzyme for plastidic isoprenoid biosynthesis in plants. J Biol Chem 276:22901-22909

Ferreres F, Figueiredo R, Bettencourt S et al (2011) Identification of phenolic compounds in isolated vacuoles of the medicinal plant Catharanthus roseus and their interaction with vacuolar class III peroxidase: $\mathrm{An}_{2} \mathrm{O}_{2}$ affair? J Exp Bot 62:2841-2854

Fischer M, Meyer S, Oswald M et al (2013) Metabolic engineering of monoterpenoid production in yeast. Isoprenoid synthesis in plants and microorganisms. Springer, New York, pp 65-71

Fukushima A, Kusano M, Redestig H et al (2009) Integrated omics approaches in plant systems biology. Curr Opin Chem Biol 13:532-538

Ganjewala D, Kumar S, Luthra R (2009) An account of cloned genes of methyl-erythritol-4-phosphate pathway of isoprenoid biosynthesis in plants. Curr Issues Mol Biol 11:i35-i45

Gantet P, Memelink J (2002) Transcription factors: tools to engineer the production of pharmacologically active plant metabolites. Trends Pharmacol Sci 23:563-569

Geerlings A, Ibañez MML, Memelink J et al (2000) Molecular cloning and analysis of strictosidine $\beta$-D-glucosidase, an enzyme in terpenoid indole alkaloid biosynthesis in Catharanthus roseus. J Biol Chem 275:3051-3056

Geerlings A, Redondo F, Contin A et al (2001) Biotransformation of tryptamine and secologanin into plant terpenoid indole alkaloids by transgenic yeast. Appl Microbiol Biotechnol 56:420-424

Geu-Flores F, Sherden NH, Courdavault V et al (2012) An alternative route to cyclic terpenes by reductive cyclization in iridoid biosynthesis. Nature 492:138-142

Glenn WS, Nims E, O'Connor SE (2011) Reengineering a tryptophan halogenase to preferentially chlorinate a direct alkaloid precursor. J Am Chem Soc 133:19346-19349

Goddijn OJ, Pennings EJ, van der Helm P et al (1995) Overexpression of a tryptophan decarboxylase cDNA in Catharanthus roseus crown gall calluses results in increased tryptamine levels but not in increased terpenoid indole alkaloid production. Transgenic Res 4:315-323

Góngora-Castillo E, Buell CR (2013) Bioinformatics challenges in de novo transcriptome assembly using short read sequences in the absence of a reference genome sequence. Nat Prod Rep 30:490-500

Góngora-Castillo E, Childs KL, Fedewa G et al (2012) Development of transcriptomic resources for interrogating the biosynthesis of monoterpene indole alkaloids in medicinal plant species. PLoS One 7:e52506

Goodbody AE, Endo T, Vukovic J et al (1988) Enzymic coupling of catharanthine and vindoline to form $3^{\prime}, 4^{\prime}$ - anhydrovinblastine by horseradish peroxidase. Planta Med 54:136-140

Guirimand G, Burlat V, Oudin A et al (2009) Optimization of the transient transformation of Catharanthus roseus cells by particle bombardment and its application to the subcellular localization of hydroxymethylbutenyl 4-diphosphate synthase and geraniol 10-hydroxylase. Plant Cell Rep 28:1215-1234

Guirimand G, Courdavault V, Lanoue A et al (2010a) Strictosidine activation in Apocynaceae: towards a "nuclear time bomb". BMC Plant Biol 10:182

Guirimand G, Courdavault V, St-Pierre B et al (2010b) Biosynthesis and regulation of alkaloids. In: Pua EC, Davey MR (eds) Plant developmental biology-biotechnological perspectives. Springer, Berlin, pp 139-160

Guirimand G, Guihur A, Ginis O et al (2011a) The subcellular organization of strictosidine biosynthesis in Catharanthus roseus epidermis highlights several trans-tonoplast translocations of intermediate metabolites. FEBS J 278:749-763

Guirimand G, Guihur A, Poutrain P et al (2011b) Spatial organization of the vindoline biosynthetic pathway in Catharanthus roseus. J Plant Physiol 168:549-557

Guirimand G, Guihur A, Phillips MA et al (2012) A single gene encodes isopentenyl diphosphate isomerase isoforms targeted to plastids, mitochondria and peroxisomes in Catharanthus roseus. Plant Mol Biol 79:443-459

Hallard DAC (2000) Transgenic plant cells for the production of indole alkaloids: PhD Thesis, Rijksuniversiteit te Leiden

Hallard DAC, Van der Heijden R, Verpoorte R et al (1997) Suspension cultured transgenic cells of Nicotiana tabacum expressing tryptophan decarboxylase and strictosidine synthase cDNAs from Catharanthus roseus produce strictosidine upon secologanin feeding. Plant Cell Rep 17:50-54

Hasunuma T, Takeno S, Hayashi S et al (2008) Overexpression of 1-deoxy-D-xylulose-5-phosphate reductoisomerase gene in chloroplast contributes to increment of isoprenoid production. J Biosci Bioeng 105:518-526

Hedhili S, Courdavault V, Giglioli-Guivarc'h N et al (2007) Regulation of the terpene moiety biosynthesis of Catharanthus roseus terpene indole alkaloids. Phytochem Rev 6:341-351

Herrmann KM, Weaver LM (1999) The shikimate pathway. Ann Rev Plant biol 50:473-503

Hong SB, Peebles CA, Shanks JV et al (2006) Expression of the Arabidopsis feedback-insensitive anthranilate synthase holoenzyme and tryptophan decarboxylase genes in Catharanthus roseus hairy roots. J Biotechnol 122:28-38

Hughes EH, Hong SB, Gibson SI et al (2004a) Expression of a feedback-resistant anthranilate synthase in Catharanthus roseus hairy roots provides evidence for tight regulation of terpenoid indole alkaloid levels. Biotechnol Bioeng 86:718-727

Hughes EH, Hong SB, Gibson SI et al (2004b) Metabolic engineering of the indole pathway in Catharanthus roseus hairy roots and increased accumulation of tryptamine and serpentine. Metab Eng 6:268-276

Irmler S, Schröder G, St-Pierre B et al (2000) Indole alkaloid biosynthesis in Catharanthus roseus: new enzyme activities and identification of cytochrome P450 CYP72A1 as secologanin synthase. Plant J 24:797-804

Jacobs DI, Gaspari M, van der Greef J et al (2005) Proteome analysis of the medicinal plant Catharanthus roseus. Planta 221:690-704 
Jaggi M, Kumar S, Sinha AK (2011) Overexpression of an apoplastic peroxidase gene $\mathrm{CrPrx}$ in transgenic hairy root lines of Catharanthus roseus. Appl Microbiol Biotechnol 90:1005-1016

Kim SW, Ban SH, Jeong SC et al (2007) Genetic discrimination between Catharanthus roseus cultivars by metabolic fingerprinting using ${ }^{1} \mathrm{H}$ NMR spectra of aromatic compounds. Biotechnol Bioprocess Eng 12:646-652

Kim HK, Choi YH, Verpoorte R (2010) NMR-based metabolomic analysis of plants. Nat Protoc 5:536-549

Kumar S, Dutta A, Sinha AK et al (2007) Cloning, characterization and localization of a novel basic peroxidase gene from Catharanthus roseus. FEBS J 274:1290-1303

Kumar S, Jaggi M, Taneja J et al (2011) Cloning and characterization of two new Class III peroxidase genes from Catharanthus roseus. Plant Physiol Biochem 49:404-412

Kumar S, Jaggi M, Sinha AK (2012) Ectopic overexpression of vacuolar and apoplastic Catharanthus roseus peroxidases confers differential tolerance to salt and dehydration stress in transgenic tobacco. Protoplasma 249:423-432

Kutchan TM (1995) Alkaloid biosynthesis-the basis for metabolic engineering of medicinal plants. Plant Cell 7:1059

Lackman P, González-Guzmán M, Tilleman S et al (2011) Jasmonate signaling involves the abscisic acid receptor PYL4 to regulate metabolic reprogramming in Arabidopsis and tobacco. Proc Natl Acad Sci USA 108:5891-5896

Laflamme P, St-Pierre B, De Luca V (2001) Molecular and biochemical analysis of a Madagascar periwinkle rootspecific minovincinine-19-hydroxy- $O$-acetyltransferase. Plant Physiol 125:189-198

Lange BM, Croteau R (1999) Isopentenyl diphosphate biosynthesis via a mevalonate-independent pathway: isopentenyl monophosphate kinase catalyzes the terminal enzymatic step. Proc Natl Acad Sci USA 96:13714-13719

Li CY, Leopold AL, Sander GW et al (2013) The ORCA2 transcription factor plays a key role in regulation of the terpenoid indole alkaloid pathway. BMC Plant Biol 13:155

Liu DH, Ren WW, Cui LJ et al (2011) Enhanced accumulation of catharanthine and vindoline in Catharanthus roseus hairy roots by overexpression of transcriptional factor ORCA2. Afr J Biotechnol 10:3260-3268

Liu LYD, Tseng HI, Lin CP et al (2014) High-throughput transcriptome analysis of the leafy flower transition of Catharanthus roseus induced by peanut witches'-broom phytoplasma infection. Plant Cell Physiol 55:942-957

Lounasmaa M, Hanhinen P (1998) Biomimetic formation and interconversion in the heteroyohimbine series. Heterocycles 48:1483-1492

Luijendijk TJ, Stevens LH, Verpoorte R (1998) Purification and characterisation of strictosidine $\beta$-D-glucosidase from Catharanthus roseus cell suspension cultures. Plant Physiol Biochem 36:419-425

Madyastha K, Coscia CJ (1979) Enzymology of indole alkaloid biosynthesis. In: Swain T, Waller GR (eds) Springer, US. pp 85-129

Magnotta M, Murata J, Chen J et al (2007) Expressionof deacetylvindoline-4- $O$-acetyltransferase in Catharanthus roseus hairy roots. Phytochemistry 68:1922-1931

Mahmoud SS, Croteau RB (2001) Metabolic engineering of essential oil yield and composition in mint by altering expression of deoxyxylulose phosphate reductoisomerase and menthofuran synthase. Proc Natl Acad Sci USA 98:8915-8920

Mahroug S, Courdavault V, Thiersault M et al (2006) Epidermis is a pivotal site of at least four secondary metabolic pathways in Catharanthus roseus aerial organs. Planta 223:1191-1200

Mahroug S, Burlat V, St-Pierre B (2007) Cellular and sub-cellular organisation of the monoterpenoid indole alkaloid pathway in Catharanthus roseus. Phytochem Rev 6:363-381

McKnight T, Roessner C, Devagupta R et al (1990) Nucleotide sequence of a cDNA encoding the vacuolar protein strictosidine synthase from Catharanthus roseus. Nucl Acids Res 18:4939

Meijer AH, Cardoso M, Voskuilen JT et al (1993a) Isolation and characterization of a cDNA clone from Catharanthus roseus encoding NADPH: cytochrome $\mathrm{P}-450$ reductase, an enzyme essential for reactions catalysed by cytochrome $\mathrm{P}-450$ mono-oxygenases in plants. Plant J 4:47-60

Meijer AH, De-Wall A, Verpoorte R (1993b) Purification of the cytochrome P-450 enzyme geraniol-10-hydroxylase from cell cultures of Catharanthus roseus. J Chromatogr 653:237-249

Menke FLH, Champion A, Kijne JW et al (1999) A novel jasmonate-and elicitor-responsive element in the periwinkle secondary metabolite biosynthetic gene STR interacts with a jasmonate-and elicitor-inducible AP2-domain transcription factor, ORCA2. EMBO J 18:4455-4463

Miettinen K, Dong L, Navrot N et al (2014) The seco-iridoid pathway from Catharanthus roseus. Nat Commun. doi:10. 1038/ncomms4606

Montiel G, Breton C, Thiersault M et al (2007) Transcription factor Agamous-like 12 from Arabidopsis promotes tissuelike organization and alkaloid biosynthesis in Catharanthus roseus suspension cells. Metab Eng 9:125-132

Moreno-Valenzuela OA, Minero-García Y, Chan W et al (2003) Increase in the indole alkaloid production and its excretion into the culture medium by calcium antagonists in Catharanthus roseus hairy roots. Biotechnol Lett 25:1345-1349

Murata J, De Luca V (2005) Localization of tabersonine 16-hydroxylase and 16-OH tabersonine-16- $O$-methyltransferase to leaf epidermal cells defines them as a major site of precursor biosynthesis in the vindoline pathway in Catharanthus roseus. Plant J 44:581-594

Murata J, Bienzle D, Brandle JE et al (2006) Expressed sequence tags from Madagascar periwinkle (Catharanthus roseus). FEBS Lett 580:4501-4507

Murata J, Roepke J, Gordon H et al (2008) The leaf epidermome of Catharanthus roseus reveals its biochemical specialization. Plant Cell 20:524-542

Mustafa NR, Verpoorte R (2007) Phenolic compounds in Catharanthus roseus. Phytochem Rev 6:243-258

Mustafa NR, Kim HK, Choi YH et al (2009a) Biosynthesis of salicylic acid in fungus elicited Catharanthus roseus cells. Phytochemistry 70:532-539

Mustafa NR, Kim HK, Choi YH et al (2009b) Metabolic changes of salicylic acid-elicited Catharanthus roseus cell suspension cultures monitored by NMR-based metabolomics. Biotechnol Lett 31:1967-1974

Newman JD, Chappell J (1999) Isoprenoid biosynthesis in plants: carbon partitioning within the cytoplasmic pathway. Crit Rev Biochem Mol Biol 34:95-106 
Noé W, Mollenschott C, Berlin J (1984) Tryptophan decarboxylase from Catharanthus roseus cell suspension cultures: purification, molecular and kinetic data of the homogenous protein. Plant Mol Biol 3:281-288

O'Connor SE (2012) Strategies for engineering plant natural products. In: Cadenas E, Packer L (eds) The iridoidderived monoterpene indole alkaloids of Catharanthus roseus. Methods in enzymology, vol 515. Elsevier Inc, pp 189-206

Okazaki Y, Shimojima M, Sawada Y et al (2009) A chloroplastic UDP-glucose pyrophosphorylase from Arabidopsis is the committed enzyme for the first step of sulfolipid biosynthesis. Plant Cell Online 21:892-909

Pan Q, Wang Q, Yuan F et al (2012) Overexpression of ORCA3 and $\mathrm{G} 10 \mathrm{H}$ in Catharanthus roseus plants regulated alkaloid biosynthesis and metabolism revealed by NMR-metabolomics. PLoS One 7:1-14

Pan Q, Dai Y, Nuringtyas TR et al (2014) Investigation of the chemomarkers correlated with flower colour in different organs of Catharanthus roseus using NMR-based metabolomics. Phytochem Anal 25:66-74

Pasquali G, Erven ASW, Ouwerkerk PBF et al (1999) The promoter of the strictosidine synthase gene from periwinkle confers elicitor-inducible expression in transgenic tobacco and binds nuclear factors GT-1 and GBF. Plant Mol Biol 39:1299-1310

Pauw B, Hilliou FAO, Martin VS et al (2004) Zinc finger proteins act as transcriptional repressors of alkaloid biosynthesis genes in Catharanthus roseus. J Biol Chem 279:52940-52948

Peebles CA, Hong SB, Gibson SI et al (2006) Effects of terpenoid precursor feeding on Catharanthus roseus hairy roots overexpressing the alpha or the alpha and beta subunits of anthranilate synthase. Biotechnol Bioeng 93:534-540

Peebles CAM, Hughes EH, Shanks JV et al (2009) Transcriptional response of the terpenoid indole alkaloid pathway to the overexpression of ORCA3 along with jasmonic acid elicitation of Catharanthus roseus hairy roots over time. Metab Eng 11:76-86

Peebles CAM, Sander GW, Hughes EH et al (2011) The expression of 1-deoxy-D-xylulose synthase and geraniol-10hydroxylase or anthranilate synthase increases terpenoid indole alkaloid accumulation in Catharanthus roseus hairy roots. Metab Eng 13:234-240

Pomahacová B, Dusek J, Dusková J et al (2009) Improved accumulation of ajmalicine and tetrahydroalstonine in Catharanthus cells expressing an ABC transporter. J Plant Physiol 166:1405-1412

Poulsen C, Bongaerts RJ, Verpoorte R (1993) Purification and characterization of anthranilate synthase from Catharanthus roseus. Eur J Biochem 212:431-440

Radwanski ER, Last RL (1995) Tryptophan biosynthesis and metabolism: biochemical and molecular genetics. Plant Cell 7:921

Rai A, Smita SS, Singh AK et al (2013) Heteromeric and homomeric geranyl diphosphate synthases from Catharanthus roseus and their role in monoterpenoid indole alkaloid biosynthesis. Mol Plant 6:1531-1549

Raina SK, Wankhede DP, Jaggi M et al (2012) CrMPK3, a mitogen activated protein kinase from Catharanthus roseus and its possible role in stress induced biosynthesis of monoterpenoid indole alkaloids. BMC Plant Biol 12:134

Ramos-Valdivia A, van der Heijden R, Verpoorte R (1997) Isopentenyl diphosphate isomerase: a core enzyme in isoprenoid biosynthesis. A review of its biochemistry and function. Nat Prod Rep 14:591-603

Ratcliffe R, Shachar-Hill Y (2005) Revealing metabolic phenotypes in plants: inputs from NMR analysis. Biol Rev 80:27-43

Ratcliffe RG, Shachar-Hill Y (2006) Measuring multiple fluxes through plant metabolic networks. Plant J 45:490-511

Rischer H, Orešič M, Seppänen-Laakso T et al (2006) Gene-tometabolite networks for terpenoid indole alkaloid biosynthesis in Catharanthus roseus cells. Proc Natl Acad Sci USA 103:5614-5619

Rodríguez-Concepción M, Ahumada I, Diez-Juez E et al (2001) 1-deoxy-D-xylulose 5-phosphate reductoisomerase and plastid isoprenoid biosynthesis during tomato fruit ripening. Plant J 27:213-222

Rohmer M (1999) The discovery of a mevalonate-independent pathway for isoprenoid biosynthesis in bacteria, algae and higher plants. Nat Prod Rep 16:565-574

Roytrakul S (2004) Transport of alkaloids and its precursor through the vacuolar membrane of Catharanthus roseus. Ph.D. Thesis. Leiden University, The Netherlands

Roytrakul S, Verpoorte R (2007) Role of vacuolar transporter proteins in plant secondary metabolism: Catharanthus roseus cell culture. Phytochem Rev 6:383-396

Runguphan W, O'Connor SE (2009) Metabolic reprogramming of periwinkle plant culture. Nat Chem Biol 5(3):151-153

Runguphan W, Maresh JJ, O'Connor SE (2009) Silencing of tryptamine biosynthesis for production of nonnatural alkaloids in plant culture. Proc Natl Acad Sci USA 106(33):13673-13678

Salim V, Yu F, Altarejos J et al (2013) Virus-induced gene silencing identifies Catharanthus roseus 7-deoxyloganic acid-7-hydroxylase, a step in iridoid and monoterpene indole alkaloid biosynthesis. Plant J 76:754-765

Schauer N, Fernie AR (2006) Plant metabolomics: towards biological function and mechanism. Trends Plant Sci 11:508-516

Schuhr CA, Radykewicz T, Sagner S et al (2003) Quantitative assessment of crosstalk between the two isoprenoid biosynthesis pathways in plants by NMR spectroscopy. Phytochem Rev 2:3-16

Shukla AK, Shasany AK, Gupta MM et al (2006) Transcriptome analysis in Catharanthus roseus leaves and roots for comparative terpenoid indole alkaloid profiles. J Exp Bot 57:3921-3932

Sibéril Y, Benhamron S, Memelink J et al (2001) Catharanthus roseus G-box binding factors 1 and 2 act as repressors of strictosidine synthase gene expression in cell cultures. Plant Mol Biol 45:477-488

Simkin AJ, Miettinen K, Claudel P et al (2012) Characterization of the plastidial geraniol synthase from Madagascar periwinkle which initiates the monoterpenoid branch of the alkaloid pathway in internal phloem associated parenchyma. Phytochemistry 85:36-43

Sriram G, González-Rivera O, Shanks JV (2006) Determination of biomass composition of Catharanthus roseus hairy roots for metabolic flux analysis. Biotechnol Prog 22:1659-1663 
Sriram G, Fulton DB, Shanks JV (2007) Flux quantification in central carbon metabolism of Catharanthus roseus hairy roots by ${ }^{13} \mathrm{C}$ labeling and comprehensive bondomer balancing. Phytochemistry 68:2243-2257

Stephanopoulos G, Stafford DE (2002) Metabolic engineering: a new frontier of chemical reaction engineering. Chem Eng Sci 57:2595-2602

Stevens LH, Giroud C, Pennings EJ et al (1993) Purification and characterization of strictosidine synthase from a suspension culture of Cinchona robusta. Phytochemistry 33:99-106

Stöckigt J, Husson H, Kan-Fan C et al (1977) Cathenamine, a central intermediate in the cell free biosynthesis of ajmalicine and related indole alkaloids. J Chem Soc Chem Commun 6:164-166

St-Pierre B, Vazquez-Flota FA, De Luca V (1999) Multicellular compartmentation of Catharanthus roseus alkaloid biosynthesis predicts intercellular translocation of a pathway intermediate. Plant Cell 11:887-900

Sumner LW, Mendes P, Dixon RA (2003) Plant metabolomics: large-scale phytochemistry in the functional genomics era. Phytochemistry 62:817-836

Supandi F, Hille S, Verpoorte R (2009) A reassessment of membrane transport and accumulation of terpenoid indole alkaloid precursors in Catharanthus roseus through mathematical modeling and simulation. MSc. Thesis Leiden University, The Netherlands

Suttipantaa N, Pattanaika S, Kulshrestha M et al (2011) The transcription factor CrWRKY1 positively regulates the terpenoid indole alkaloids biosynthesis in Catharanthus roseus. Plant Physiol 157:2081-2093

Toki K, Saito N, Irie Y et al (2008) 7-O-Methylated anthocyanidin glycosides from Catharanthus roseus. Phytochemistry 69:1215-1219

Urbanczyk-Wochniak E, Luedemann A, Kopka J et al (2003) Parallel analysis of transcript and metabolic profiles: a new approach in systems biology. EMBO Rep 4:989-993

van der Fits L, Memelink J (2000) ORCA3, a jasmonate-responsive transcriptional regulator of plant primary and secondary metabolism. Science 289:295-297

van der Fits L, Memelink J (2001) The jasmonate-inducible AP2/ERF-domain transcription factor ORCA3 activates gene expression via interaction with a jasmonate-responsive promoter element. Plant J 25:43-53

van der Fits L, Zhang H, Menke FLH et al (2000) A Catharanthus roseus $\mathrm{BPF}-1$ homologue interacts with an elicitorresponsive region of the secondary metabolite biosynthetic gene Str and is induced by elicitor via a JA-independent signal transduction pathway. Plant Mol Biol 44:675-685

van der Heijden R, Jacobs DI, Snoeijer W et al (2004) The Catharanthus alkaloids: pharmacognosy and biotechnology. Curr Med Chem 11:607-628

Van Moerkercke A, Fabris M, Pollier J et al (2013) CathaCyc, a metabolic pathway database built from Catharanthus roseus RNA-Seq data. Plant Cell Physiol 54:673-685

Veau B, Courtois M, Oudin A et al (2000) Cloning and expression of cDNAs encoding two enzymes of the MEP pathway in Catharanthus roseus. BBA-Gene Struct Express 1517:159-163
Verma P, Mathur AK, Srivastava A et al (2012) Emerging trends in research on spatial and temporal organization of terpenoid indole alkaloid pathway in Catharanthus roseus: a literature update. Protoplasma 249:255-268

Verma M, Ghangal R, Sharma R et al (2014) Transcriptome analysis of Catharanthus roseus for gene discovery and expression profiling. PLoS One 9:e103583

Verpoorte R, Alfermann AW (2000) Metabolic engineering of plant secondary metabolism. Kluwer Academic Publishers, Dordrecht

Verpoorte R, Van der Heijden R, Moreno PR (1997) Biosynthesis of terpenoid indole alkaloids in Catharanthus roseus cells. Alkaloids Chem Pharmacol 49:221-299

Wang CT, Liu H, Gao XS et al (2010) Overexpression of $G 10 H$ and ORCA3 in the hairy roots of Catharanthus roseus improves catharanthine production. Plant Cell Rep 29:887-894

Wang Q, Xing S, Pan Q et al (2012) Development of efficient Catharanthus roseus regeneration and transformation system using agrobacterium tumefaciens and hypocotyls as explants. BMC Biotechnol 12:34

Whitmer S, van der Heijden R, Verpoorte R (2002a) Effect of precursor feeding on alkaloid accumulation by a strictosidine synthase over-expressing transgenic cell line S1 of Catharanthus roseus. Plant Cell Tissue Organ Cult 69:85-93

Whitmer S, van der Heijden R, Verpoorte R (2002b) Effect of precursor feeding on alkaloid accumulation by a tryptophan decarboxylase over-expressing transgenic cell line T22 of Catharanthus roseus. J Biotechnol 96:193-203

Yamamoto H, Katano N, Ooi A et al (2000) Secologanin synthase which catalyzes the oxidative cleavage of loganin into secologanin is a cytochrome $\mathrm{P} 450$. Phytochemistry 53:7-12

Yang SO, Kim SH, Kim Y et al (2009) Metabolic discrimination of Catharanthus roseus calli according to their relative locations using ${ }^{1} \mathrm{H}-\mathrm{NMR}$ and principal component analysis. Biosci Biotechnol Biochem 73:2032-2036

Yu F, De Luca V (2013) ATP-binding cassette transporter controls leaf surface secretion of anticancer drug components in Catharanthus roseus. Proc Natl Acad Sci USA 24:110-139

Zhang XH, Brotherton JE, Widholm JM et al (2001) Targeting a nuclear anthranilate synthase $\alpha$-subunit gene to the tobacco plastid genome results in enhanced tryptophan biosynthesis. Return of a gene to its pre-endosymbiotic origin. Plant Physiol 127:131-141

Zhang H, Hedhili S, Montiel G et al (2011) The basic helix-loophelix transcription factor CrMYC2 controls the jasmonateresponsive expression of the ORCA genes that regulate alkaloid biosynthesis in Catharanthus roseus. Plant $\mathrm{J}$ 67:61-71

Zhao J, Hu Q, Guo YQ et al (2001) Elicitor-induced indole alkaloid biosynthesis in Catharanthus roseus cell cultures is related to $\mathrm{Ca}^{2+}$ influx and the oxidative burst. Plant Sci 161:423-431

Zhou ML, Hou HL, Zhu XM et al (2011) Soybean transcription factor GmMYBZ2 represses catharanthine biosynthesis in hairy roots of Catharanthus roseus. Appl Microbiol Biotechnol 91:1095-1105 